\title{
Appraisement of planar, bending and twisting cracks in 3D with isotropic and orthotropic damage models
}

\author{
G. B. Barbat, M. Cervera and M. Chiumenti \\ International Center for Numerical Methods in Engineering (CIMNE) \\ Technical University of Catalonia - BarcelonaTECH \\ Edificio C1, Campus Norte, Jordi Girona 1-3 \\ 08034 Barcelona, Spain \\ gbarbat@cimne.upc.edu, miguel.cervera@upc.edu, michele@cimne.upc.edu
}

\begin{abstract}
This paper discusses the modeling of cracking in quasi-brittle materials using isotropic and orthotropic damage constitutive laws. A mixed strain/displacement finite element formulation is used, taking advantage of its enhanced precision and its enforced interelemental strain continuity. On the one hand, this formulation avoids the spurious mesh dependency of the computed solution associated to standard elements and does not require the use of tracking techniques. On the other hand, it greatly alleviates the spurious stress locking associated to the use of orthotropic models on standard finite elements.

The performance of several isotropic and orthotropic damage constitutive laws is assessed through an extensive comparison with analytical solutions, numerical tests and experimental evidence reported in the literature. The behavior of the different damage models in terms of crack surface, collapse mechanism and force displacement curves is investigated performing 3D analyses in several conditions including Mode I, Mixed Mode and Mode III fracture.

When performing the appraisement of planar, bending and twisting cracks, the enhanced accuracy of the mixed formulation allows for a distinct assessment of the several damage models considered. Aspects related to the behavior of damage models, such as the influence of Poisson's ratio, the shape of the damage surface and the adoption of isotropic and orthotropic models are investigated and noteworthy conclusions are drawn.
\end{abstract}

Keywords: Damage, Isotropy, Orthotropy, Constitutive law, Cracking, Mixed Finite Elements, Strain Localization 


\section{Introduction}

Classical orthotropic crack models developed in the early 1970s for the modeling of cracking in concrete were largely abandoned in the late 1990s due, among others, to issues of spurious stress locking that made their use unreliable [1, 2]. Fixed Crack [3] and Rotating Crack Models [4, 5], as well as Multiple Fixed Crack [6,7] and Microplane Models [8, 9], were proposed in the three in-between decades. For more details on those crack models the review of references [1, 10-15] is suggested. Spurious stress transfer brings in spurious energy dissipation and the associated stress locking during the cracking process and hamstrings the formation of realistic failure mechanisms. These serious hindrances are partly avoided by adopting isotropic damage models, where the inadequacy of the kinematical description of standard finite elements does not show in the stress field [2, 16-19]. Nowadays, isotropic damage has been adopted as a standard practice in the modeling of quasi-brittle materials.

Regretfully, the adoption of isotropic models is far from solving the problem of FE modeling of cracking. On the one hand, it is well established that the standard FE formulation produces meshbiased results in many situations, due to its local lack of convergence in quasi-singular situations $[20,21]$. On the other hand, an isotropic description of damage is not adequate for certain applications. For example, orthotropic models are needed in cyclic loading problems to take microcrack closure-reopening (MCR) effects into account [22].

Recently, mixed finite elements have been reexamined by [20, 21, 23-25] to deal with cracking problems. Mixed finite element formulations have proved to be a remedy for spurious mesh

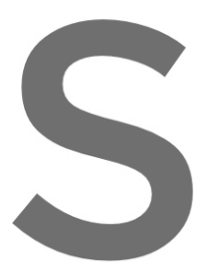
dependency and ladk elements. The use improvement over : fields accuracy both approximated independently from the displace
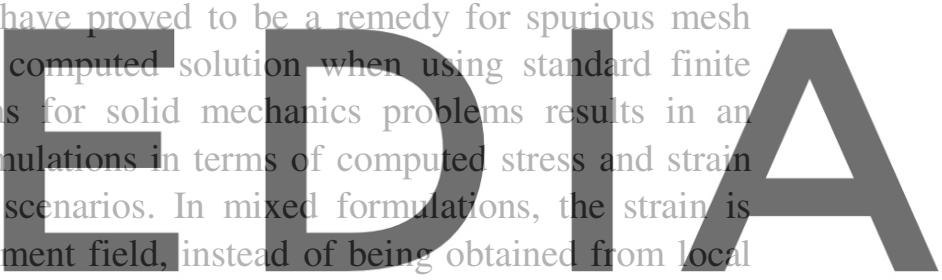

discrete differentiation at element level. In this way, more accurate stress and strain fields are

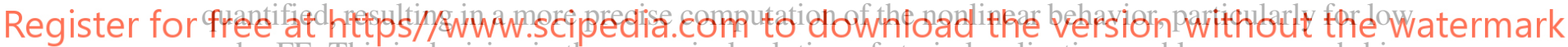
order FE. This is decisive in the numerical solution of strain localization problems, as mesh-bias independent outcomes are obtained without the need of auxiliary crack tracking techniques.

The mixed finite element technology has proved to be able to solve many of the problems related to standard elements. In [25], quasi-brittle cracks were modelled in 2D and 3D using several isotropic models. The use of an independent approximation for the strain field enforces the continuity of strains in the computed solution, whereas strains are inter-element discontinuous in standard FE. Strain continuity is crucial in alleviating the spurious stress locking that made the use of orthotropic models unpractical in the past decades. The enhanced accuracy of mixed finite elements allows now to reconsider the use of orthotropic damage constitutive laws in cracking models.

Therefore, the objectives of this paper are: (1) to assess the performance of several isotropic and orthotropic damage models to solve cracking problems in mode I, mixed mode and mode III loading, (2) to show that different models, and even the isotropic and orthotropic versions of the same model, produce different crack patterns for a given loading, (3) to demonstrate the capability of the mixed finite element formulation in successfully incorporating orthotropic damage models, as a consequence of their enforced strain continuity and enhanced accuracy. To attain these objectives, an exhaustive validation of the models is performed using theoretical results and experimental data from the literature. 
The outline of this paper is as follows: in section 2, the isotropic and orthotropic damage constitutive laws considered in this article are presented, to be used in conjunction with the mixed finite element formulation summarized in section 3. Section 4 presents numerical simulations performed in $3 \mathrm{D}$ where the performance of the constitutive laws is examined. Finally, the conclusions of the study are presented.

\section{Isotropic and orthotropic constitutive damage models}

In this section, the several constitutive damage models considered in this article are described. Using Voigt's convention, the strain and stress tensors, $\boldsymbol{\varepsilon}$ and $\boldsymbol{\sigma}$, are expressed as vectors [26]. In 3D analysis the strain vector $\varepsilon=\left(\varepsilon_{x}, \varepsilon_{y}, \varepsilon_{z}, \gamma_{x y}, \gamma_{y z}, \gamma_{x z}\right)^{T}$ has 6 components. Correspondingly, the stress $\boldsymbol{\sigma}$ is also a vector with 6 components, $\boldsymbol{\sigma}=\left(\sigma_{x}, \sigma_{y}, \sigma_{z}, \tau_{x y}, \tau_{y z}, \tau_{x z}\right)^{T}$. The stress $\boldsymbol{\sigma}$ and the strain $\varepsilon$ are linked through the constitutive equation:

$$
\sigma=\mathbf{D}_{S} \varepsilon
$$

where $\mathbf{D}_{s}$ is the secant constitutive matrix, which has to be symmetric and positive semidefinite from thermodynamic considerations. In damage models $\mathbf{D}_{S}$ is a function of a set of internal variables $d$ that describe the degradation of the material such that
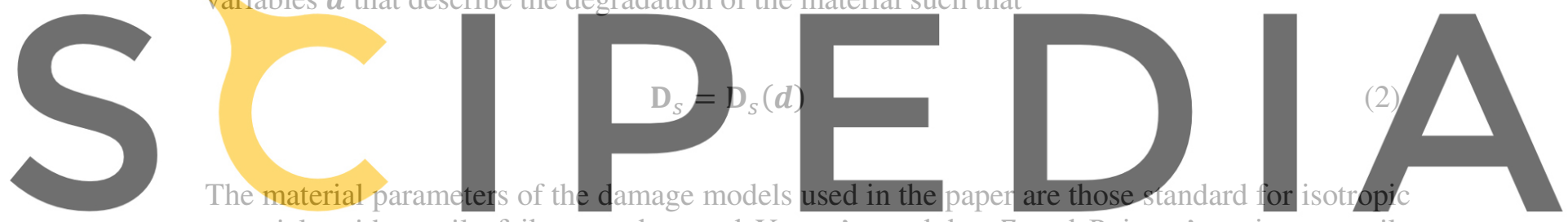

materials with tensile failure: undamaged Young's modulus $E$ and Poisson's ratio v, tensile

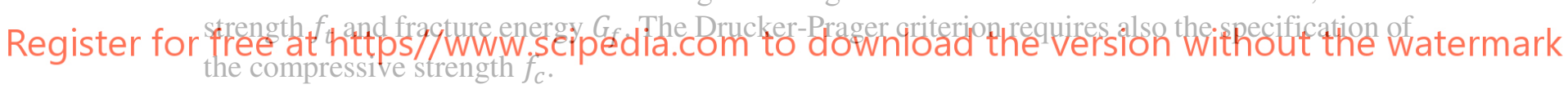

\subsection{Isotropic damage models}

In isotropic damage, the secant constitutive matrix $\mathbf{D}_{s}$ of equation (2) can be written as

$$
\mathbf{D}_{\mathrm{s}}=(1-d) \mathbf{D}_{0}=(1-d)\left(\begin{array}{ccccccc}
D_{11} & D_{12} & D_{13} & & & \\
D_{21} & D_{22} & D_{23} & & \mathbf{0} & \\
D_{31} & D_{32} & D_{33} & & & \\
& & & G_{12} & & \\
& \mathbf{0} & & & G_{13} & \\
& & & & & G_{23}
\end{array}\right)
$$

where $d$ is the internal damage index and $\mathbf{D}_{0}$ is the elastic constitutive matrix for homogeneous materials such that

$$
\begin{gathered}
D_{11}=D_{22}=D_{33}=\frac{E(1-v)}{(1+v)(1-2 v)} \\
D_{12}=D_{21}=D_{13}=D_{31}=D_{23}=D_{32}=\frac{E v}{(1+v)(1-2 v)} \\
G_{12}=G_{13}=G_{23}=\frac{E}{2(1+v)}
\end{gathered}
$$


where $E$ and $v$ are the undamaged elastic values of Young's modulus and Poisson's ratio respectively.

The damage index $d$ is an internal variable that measures the loss of stiffness of the material and it ranges $0 \leq d \leq 1$.

From equations (1) and (3), the constitutive equation of an isotropic damage model can be written as

$$
\boldsymbol{\sigma}=(1-d) \mathbf{D}_{0} \boldsymbol{\varepsilon}=(1-d) \overline{\boldsymbol{\sigma}}
$$

where the effective stress $\overline{\boldsymbol{\sigma}}$ is introduced as $\overline{\boldsymbol{\sigma}}=\mathbf{D}_{0} \boldsymbol{\varepsilon}$, corresponding to the hypothesis of strain equivalence [21].

The damage criterion, $\mathbb{F}$, is defined as

$$
\mathbb{F}\left(\sigma_{e q}, r\right)=\sigma_{e q}(\bar{\sigma})-r \leq 0
$$

where $\sigma_{e q}(\overline{\boldsymbol{\sigma}})$ is the equivalent effective stress, and $r$ is the current stress-like damage threshold. For tensile cracking the initial value of the damage threshold is taken as the tensile strength of the material, $r_{0}=f_{t}$.
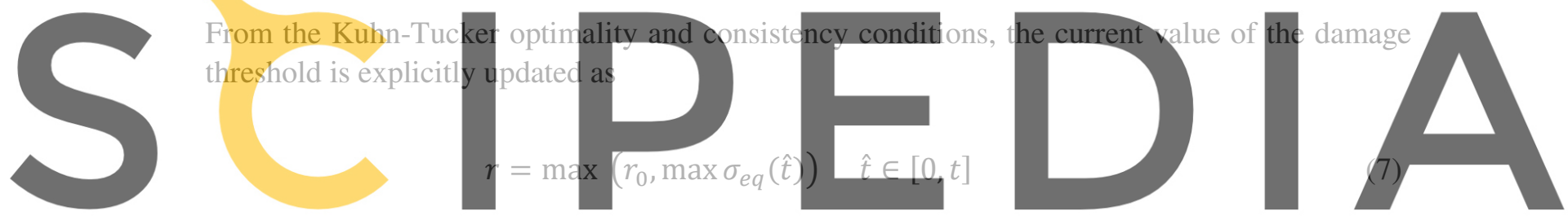

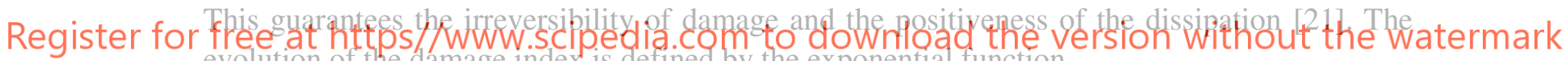
evolution of the damage index is defined by the exponential function

$$
d=d(r)=1-\frac{r_{0}}{r} \exp \left(-2 H_{S}\left(\frac{\left\langle r-r_{0}\right\rangle}{r_{0}}\right)\right)
$$

where $H_{S}$ is the positive softening parameter, which controls the rate of material degradation.

In FE simulations of quasi-brittle failure [20, 24] following the smeared (or crack band) approach, the softening parameter is linked to the material fracture energy $G_{f}$, which is a property of the material, in the following way:

$$
H_{S}=\frac{\bar{H}_{S} b}{1-\bar{H}_{S} b}
$$

where $b$ is the bandwidth of the smeared crack and $\bar{H}_{S}$ is the inverse of the material length $\mathcal{L}$

$$
\bar{H}_{S}^{-1}=\mathcal{L}=\frac{2 E G_{f}}{\left(f_{t}\right)^{2}}
$$

$f_{t}$ being the tensile strength and $E$ the Young's modulus. In this work, the bandwidth of the localized cracks is taken as 


$$
b=\left(1-\tau_{\varepsilon}\right) 2 h+\tau_{\varepsilon} h=\left(2-\tau_{\varepsilon}\right) h
$$

where $h$ is the finite element size and $\tau_{\varepsilon}$ is the stabilization parameter with value $0 \leq \tau_{\varepsilon} \leq 1$. This is consistent with the approximation adopted for the discrete strain field in the mixed formulation, see equation (38).

\section{Damage criteria}

This article centers in the assessment of the relative performance of several damage models. Consequently, the effective equivalent stress $\sigma_{e q}(\overline{\boldsymbol{\sigma}})$ is defined through different criteria. Specifically, the Beltrami, Modified Beltrami, Positive Beltrami, Rankine and Drucker-Prager criteria are considered.

- Beltrami

$$
\sigma_{e q}(\bar{\sigma})=\sqrt{\left(\bar{\sigma}_{1}\right)^{2}+\left(\bar{\sigma}_{2}\right)^{2}+\left(\bar{\sigma}_{3}\right)^{2}-2 v\left(\bar{\sigma}_{1} \bar{\sigma}_{2}+\bar{\sigma}_{1} \bar{\sigma}_{3}+\bar{\sigma}_{2} \bar{\sigma}_{3}\right)}
$$

where $\bar{\sigma}_{1}, \bar{\sigma}_{2}$ and $\bar{\sigma}_{3}$ are the major principal stresses and $v$ is the Poisson ratio.
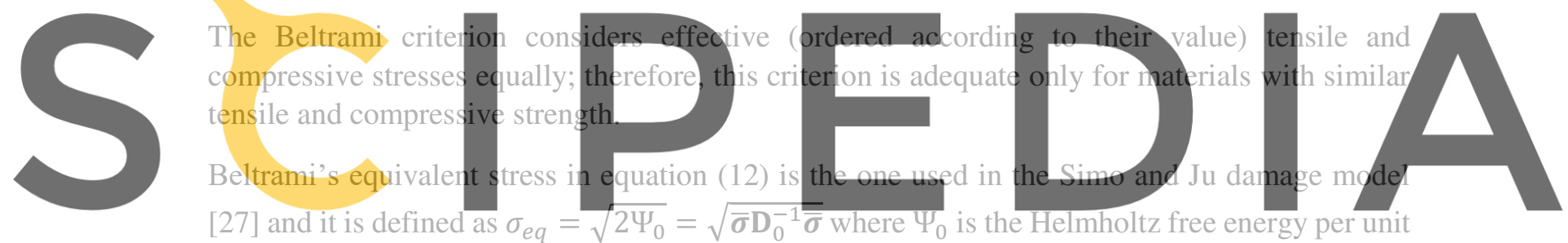

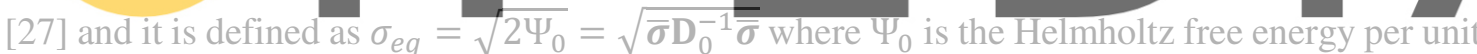

Register for free at https//WWW.scipedia.com to download the version without the watermark

The criterion is similar to the well-known Von Mises criterion, where

$$
\sigma_{e q}(\overline{\boldsymbol{\sigma}})=\sqrt{\left(\bar{\sigma}_{1}\right)^{2}+\left(\bar{\sigma}_{2}\right)^{2}+\left(\bar{\sigma}_{3}\right)^{2}-\left(\bar{\sigma}_{1} \bar{\sigma}_{2}+\bar{\sigma}_{1} \bar{\sigma}_{3}+\bar{\sigma}_{2} \bar{\sigma}_{3}\right)}
$$

but is not purely isochoric.

\section{- Positive Beltrami}

$$
\sigma_{e q}(\overline{\boldsymbol{\sigma}})=\sqrt{\left(\left\langle\bar{\sigma}_{1}\right\rangle\right)^{2}+\left(\left\langle\bar{\sigma}_{2}\right\rangle\right)^{2}+\left(\left\langle\bar{\sigma}_{3}\right\rangle\right)^{2}-2 v\left(\left\langle\bar{\sigma}_{1}\right\rangle\left\langle\bar{\sigma}_{2}\right\rangle+\left\langle\bar{\sigma}_{1}\right\rangle\left\langle\bar{\sigma}_{3}\right\rangle+\left\langle\bar{\sigma}_{2}\right\rangle\left\langle\bar{\sigma}_{3}\right\rangle\right)}
$$

where $\langle\cdot\rangle$ are the Macaulay brackets, such that $\langle x\rangle=x$ if $x \geq 0,0$ if $x<0$.

This criterion is introduced to consider only damage under tensile (positive) stresses, so that damage is driven by the positive part of the Helmholtz free energy $\sigma_{e q}=\sqrt{2 \Psi_{0}^{+}}=\sqrt{\overline{\boldsymbol{\sigma}}^{+} \mathbf{D}_{0}^{-1} \overline{\boldsymbol{\sigma}}^{+}}$ being $\overline{\boldsymbol{\sigma}}^{+}$the vector that contains the positive part of the effective stresses, $\overline{\boldsymbol{\sigma}}^{+}=$ $\left(\begin{array}{llllll}\left\langle\bar{\sigma}_{1}\right\rangle & \left\langle\bar{\sigma}_{2}\right\rangle & \left\langle\bar{\sigma}_{3}\right\rangle & 0 & 0 & 0\end{array}\right)^{T}$. 


\section{- Modified Beltrami}

A modification of the Beltrami model is considered in reference [28], in which Poisson's effect is accounted for differently in the evaluation of the equivalent stress and the computation of the constitutive matrix. The equivalent stress is

$$
\sigma_{e q}(\overline{\boldsymbol{\sigma}})=\sqrt{\left(\bar{\sigma}_{1}\right)^{2}+\left(\bar{\sigma}_{2}\right)^{2}+\left(\bar{\sigma}_{3}\right)^{2}}
$$

The secant constitutive matrix is similar to that in equation (3):

$$
\begin{gathered}
D_{11}=D_{22}=D_{33}=\frac{E(1-\tilde{v})}{(1+\tilde{v})(1-2 \tilde{v})} \\
D_{12}=D_{21}=D_{13}=D_{31}=D_{23}=D_{32}=\frac{E \tilde{v}}{(1+\tilde{v})(1-2 \tilde{v})} \\
G_{12}=G_{13}=G_{23}=\frac{E}{2(1+\tilde{v})}
\end{gathered}
$$

and the term $\tilde{v}$ is defined as $\tilde{v}=(1-d) v$.

\section{A modified positive Beltrami criterion can also be defined.}

\section{- Rankine}
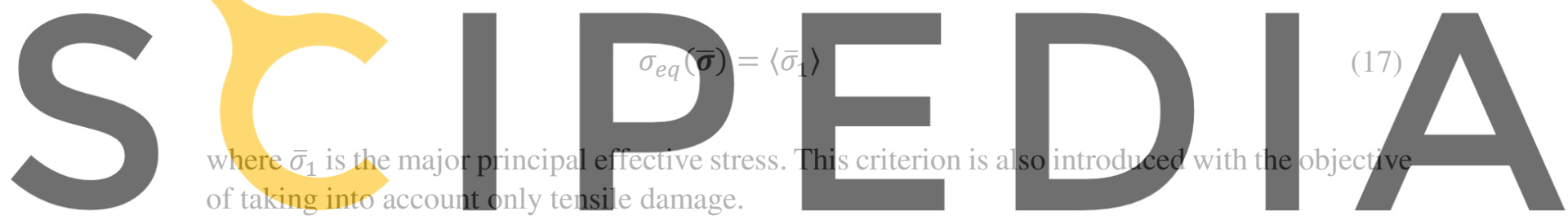

Register for free at Ducker-Prager

$$
\begin{aligned}
& \text { v.scipedia.com to download the } \\
& \sigma_{e q}(\bar{\sigma})=\frac{3}{3+\tan \phi}\left(\sqrt{3 J_{2}}+\tan \phi \frac{I_{1}}{3}\right)
\end{aligned}
$$

where $I_{1}$ and $J_{2}$ are the first and second effective stress invariants, which are expressed in terms of the principal stresses as

$$
\begin{gathered}
I_{1}=\left(\bar{\sigma}_{1}+\bar{\sigma}_{2}+\bar{\sigma}_{3}\right) \\
J_{2}=\frac{1}{6}\left[\left(\bar{\sigma}_{1}-\bar{\sigma}_{2}\right)^{2}+\left(\bar{\sigma}_{2}-\bar{\sigma}_{3}\right)^{2}+\left(\bar{\sigma}_{3}-\bar{\sigma}_{1}\right)^{2}\right]
\end{gathered}
$$

and $\phi$ is the internal friction angle of the material. The friction angle $\phi$ can be related to the uniaxial tensile and compressive strengths, $f_{t}$ and $f_{c}$, as

$$
\tan \phi=3 \frac{f_{c}-f_{t}}{f_{c}+f_{t}}
$$

This criterion is appropriate for materials with different strengths for traction and compression and subjected to mixed mode loading. 


\subsection{Orthotropic damage models}

In this article, orthotropic damage models with 3 independent damage indices, one for each principal direction of effective strain and stress, are also considered. Such symmetric orthotropic models are formulated using the hypothesis of energy equivalence [22, 29-31]. Equation (2) can be particularized for the orthotropic damage models as

$$
\begin{aligned}
& \mathbf{D}_{s}=\mathbf{D}_{s}(\boldsymbol{d})=
\end{aligned}
$$

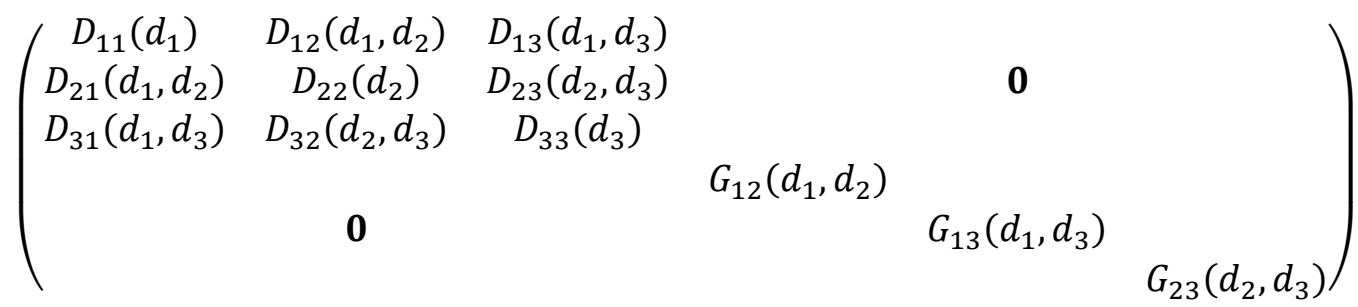

such that

$$
D_{i i}=\left(1-d_{i}\right) \frac{E(1-v)}{(1+v)(1-2 v)} \quad ; \quad i=1,3
$$$$
D_{i j}=\sqrt{\left(1-d_{i}\right)\left(1-d_{j}\right)} \frac{E v}{(1+v)(1-2 v)} \quad ; \quad i, j=1,3 \quad i \neq j
$$
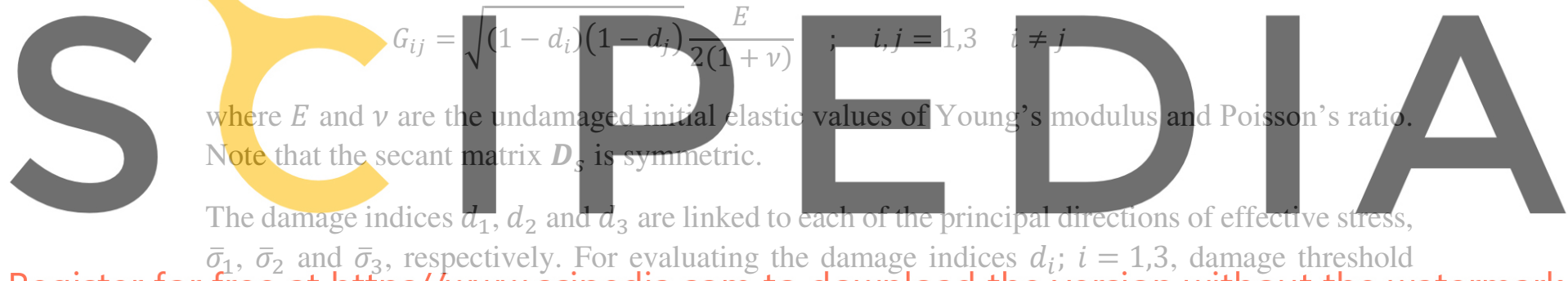

$\bar{\sigma}_{1}, \bar{\sigma}_{2}$ and $\bar{\sigma}_{3}$, respectively. For evaluating the damage indices $d_{i} ; i=1,3$, damage threshold

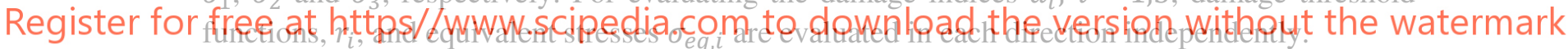

The damage criteria are

$$
\mathbb{F}_{i}\left(\sigma_{e q, i}, r_{i}\right)=\sigma_{e q, i}(\overline{\boldsymbol{\sigma}})-r_{i} \leq 0
$$

Damage thresholds are evaluated as

$$
r_{i}=\max \left(r_{0}, \max \sigma_{e q, i}(\hat{t})\right) \quad \hat{t} \in[0, t]
$$

similarly to equation (7). The corresponding damage indices $d_{i}$ are calculated as

$$
d_{i}=d_{i}\left(r_{i}\right)=1-\frac{r_{0}}{r_{i}} \exp \left(-2 H_{S}\left(\frac{\left\langle r_{i}-r_{0}\right\rangle}{r_{0}}\right)\right)
$$

where $H_{S}$ is the parameter evaluated according to equations (9)-(11).

The orthotropic model laid out here falls within the rotating crack approach; "fixed" orthotropic damage models may also be considered [22]. 
The equivalent stress in each direction $\sigma_{e q, i}(\overline{\boldsymbol{\sigma}})$, according to the several criteria, are

\section{- Beltrami}

$$
\begin{gathered}
\sigma_{e q, 1}=\sqrt{\left(\bar{\sigma}_{1}\right)^{2}+\left(\bar{\sigma}_{2}\right)^{2}+\left(\bar{\sigma}_{3}\right)^{2}-2 v\left(\bar{\sigma}_{1} \bar{\sigma}_{2}+\bar{\sigma}_{1} \bar{\sigma}_{3}+\bar{\sigma}_{2} \bar{\sigma}_{3}\right)} \\
\sigma_{e q, 2}=0 \\
\sigma_{e q, 3}=0
\end{gathered}
$$

so that damage affects only the direction of the maximum principal stress.

- Rankine

$$
\sigma_{e q, i}=\left\langle\bar{\sigma}_{i}\right\rangle
$$

Like in its isotropic form, the orthotropic Rankine damage model is only sensitive to tensile stresses, but considers each principal direction indepently.

\section{Drucker-Prager}

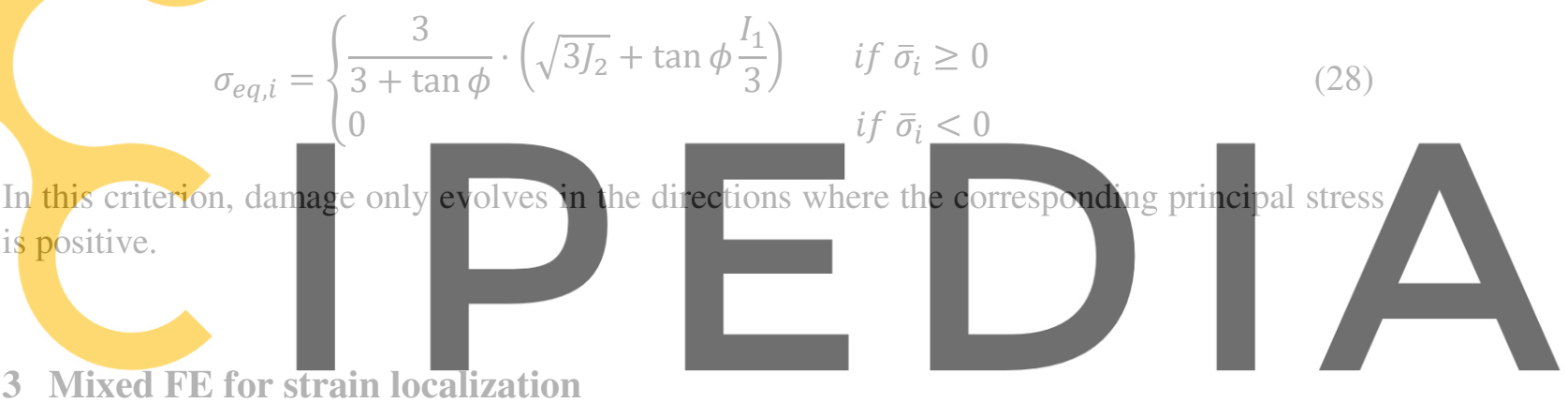

\section{Mixed FE for strain localization}

\section{Register for free at https//www.scipedia.com to download the version without the watermark}

In this section, the adopted mixed finite element formulation is introduced. This formulation is presented in detail in reference [25]. For more details, references [20, 21, 24, 32] are suggested.

The mixed finite element formulation here presented fits into the continuous approach, as the crack is represented at constitutive level using the damage models detailed in Section 2. Therefore, the separation between the two opposite sides of the crack is modelled through continuous (linear) displacement and strain fields [25].

In the considered mixed FE formulation, the variational form of the nonlinear solid mechanics problem is cast in terms of the displacement $\boldsymbol{u}$ and the strain $\boldsymbol{\varepsilon}$ fields. Matrix and vector notation based on Voigt's convention for symmetric tensors is adopted, as customarily used in FE literature and in codes. Writing the problem in matrix form, $\boldsymbol{u}$ and $\boldsymbol{\varepsilon}$ are expressed in Voigt's convention as vectors. Details of the algebraic system of equations are given in [25].

The strain and displacement fields are locally related through the compatibility equation [26]

$$
\varepsilon=\boldsymbol{S u}
$$

where $\mathcal{S}$ is the differential symmetric gradient operator. Correspondingly, the stress vector $\boldsymbol{\sigma}$ and the body forces vector $\mathbf{f}$ are linked through Cauchy's equilibrium equation of a body, written in matrix form as 


$$
\boldsymbol{S}^{T} \boldsymbol{\sigma}+\mathbf{f}=\mathbf{0}
$$

where $\boldsymbol{S}^{T}$ is the differential divergence operator, adjoint to the $\boldsymbol{S}$ in (29). The stress vector $\boldsymbol{\sigma}$ and the strain vector $\boldsymbol{\varepsilon}$ are linked by the constitutive equation:

$$
\boldsymbol{\sigma}=\mathbf{D}_{s} \boldsymbol{\varepsilon}
$$

where $\mathbf{D}_{s}$ is the secant constitutive matrix.

Pre-multiplying equation (29) by the secant constitutive matrix $\mathbf{D}_{s}$ and substituting equation (31) into equation (30) results in the mixed system of equations

$$
\begin{gathered}
-\mathbf{D}_{S} \varepsilon+\mathbf{D}_{S} \boldsymbol{s} \boldsymbol{u}=\mathbf{0} \\
\mathcal{S}^{T}\left(\mathbb{D}_{S} \varepsilon\right)+\mathfrak{f}=0
\end{gathered}
$$

The system of equations (32)-(33) is the strong form of the mixed $\boldsymbol{\varepsilon} / \boldsymbol{u}$ formulation, completed with the proper boundary conditions.
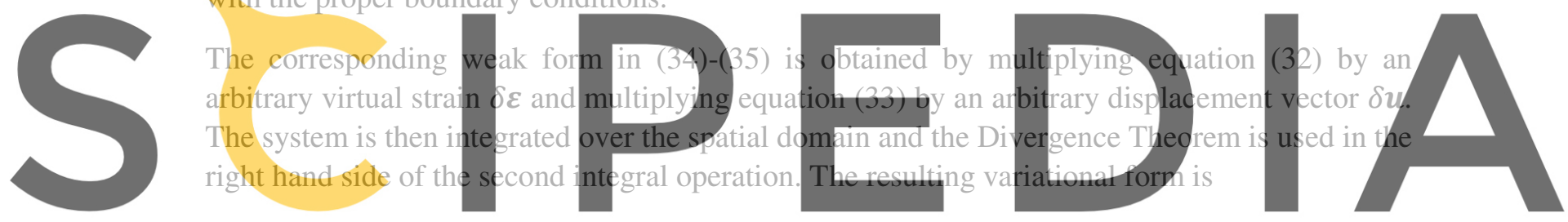

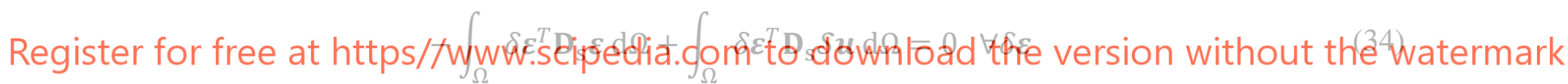

$$
\int_{\Omega}(\boldsymbol{\delta} \delta \boldsymbol{u})^{T}\left(\mathbf{D}_{s} \boldsymbol{\varepsilon}\right) \mathrm{d} \Omega=\int_{\Omega} \delta \boldsymbol{u}^{T} \mathbf{f} \mathrm{d} \Omega+\int_{\Gamma_{t}} \delta \boldsymbol{u}^{T} \overline{\boldsymbol{t}} \mathrm{d} \Gamma \quad \forall \delta \boldsymbol{u}
$$

The mixed problem to be solved is to find the unknowns $\boldsymbol{u}$ and $\boldsymbol{\varepsilon}$ that verify the system of equations composed by (34) and (35) and that verify the boundary condition $\boldsymbol{u}=\mathbf{0}$ on $\Gamma_{u}$ given the arbitrary virtual displacement $\delta \boldsymbol{u}$, which vanishes on the Dirichlet boundary $\Gamma_{u}$ and arbitrary virtual strain $\delta \boldsymbol{\varepsilon}$. Note that this variational problem is symmetric if $\mathbf{D}_{\mathrm{s}}$ is symmetric.

The FE discrete form of the mixed problem is obtained by discretizing the domain in FE, so that $\Omega=\cup \Omega_{e}$, and substituting the displacement $\boldsymbol{u}$ and the strain $\boldsymbol{\varepsilon}$ with the FE discrete approximations $\widehat{\boldsymbol{u}}$ and $\hat{\boldsymbol{\varepsilon}}$ defined element-wise as

$$
\begin{aligned}
& \boldsymbol{u} \cong \widehat{\boldsymbol{u}}=\boldsymbol{N}_{u} \mathrm{U} \\
& \varepsilon \cong \widehat{\varepsilon}=N_{\varepsilon} E
\end{aligned}
$$


where $\boldsymbol{U}$ and $\mathbf{E}$ are vectors containing the values of the displacements and the strains at the nodes of the finite element mesh. $\boldsymbol{N}_{u}$ and $\boldsymbol{N}_{\varepsilon}$ are the matrices containing the interpolation functions adopted in the FE approximation.

To ensure solvability (i.e. uniqueness) and stability of the solution in the system of equations, the interpolation functions in (36)-(37) must satisfy the Inf-Sup condition. This requirement is not fulfilled if equal interpolations are used for strains and displacements. In such case, the solution is unstable, and spurious oscillations may appear in the discrete displacement field. To be able to circumvent the strictness of the Inf-Sup condition and to use linear approximations both for displacements and strains a stabilization procedure is necessary to provide stability to the mixed discrete formulation. The stabilization procedure consists in the modification of the discrete variational form using the Orthogonal Subscales Method, introduced within the framework of the Variational Multiscale Stabilization methods and adopted herein.

The basic idea of the stabilization procedure is to substitute the approximation of the discrete strain in equation (37) by the following stabilized discrete field

$$
\boldsymbol{\varepsilon} \cong \hat{\varepsilon}=N_{\varepsilon} \boldsymbol{E}+\tau_{\varepsilon}\left(\boldsymbol{B}_{u} \boldsymbol{U}-\boldsymbol{N}_{\varepsilon} \boldsymbol{E}\right)=\left(1-\tau_{\varepsilon}\right) \boldsymbol{N}_{\varepsilon} \boldsymbol{E}+\tau_{\varepsilon} \boldsymbol{B}_{u} \boldsymbol{U}
$$

where $\tau_{\varepsilon}$ is a stabilization parameter with value $0 \leq \tau_{\varepsilon} \leq 1$. Note that for $\tau_{\varepsilon}=1$, the strain interpolation of the standard irreducible formulation is recovered:
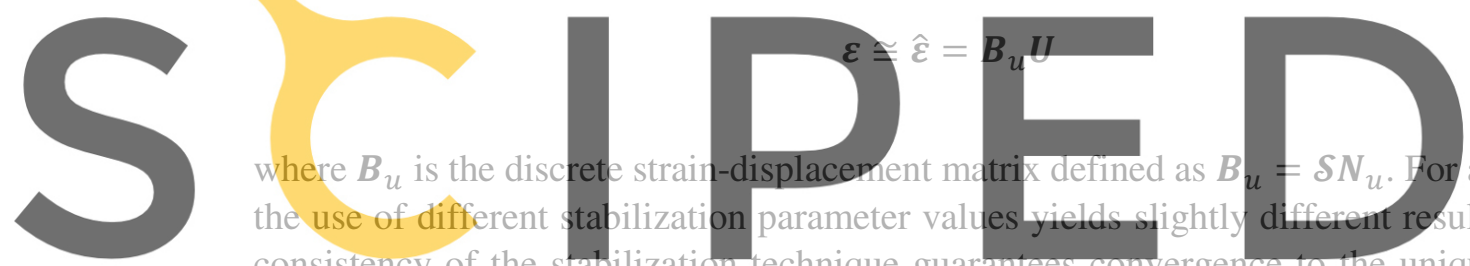

consistency of the stat

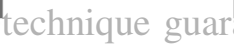

rgentection

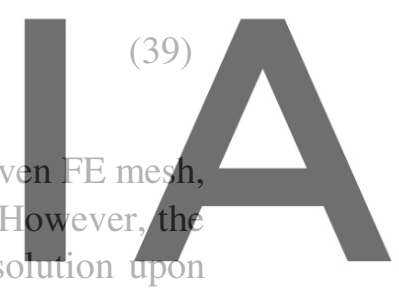

mesh refinement. Additional details are given in reference [25.

Register for free at https//www.scipedia.com to download the version without the watermark The resulting algebraic system of equations reads:

$$
\left[\begin{array}{cc}
-M_{\tau} & G_{\tau} \\
G_{\tau}^{T} & K_{\tau}
\end{array}\right]\left[\begin{array}{l}
E \\
U
\end{array}\right]=\left[\begin{array}{l}
\mathbf{0} \\
F
\end{array}\right]
$$

such that $\boldsymbol{M}_{\boldsymbol{\tau}}=\left(1-\tau_{\varepsilon}\right) \boldsymbol{M}, \boldsymbol{G}_{\boldsymbol{\tau}}=\left(1-\tau_{\varepsilon}\right) \boldsymbol{G}$ and $\boldsymbol{K}_{\boldsymbol{\tau}}=\tau_{\varepsilon} \boldsymbol{K}$ with

$$
\begin{gathered}
\boldsymbol{M}=\int_{\Omega} \boldsymbol{N}_{\varepsilon}^{T} \mathbf{D}_{\mathbf{s}} \boldsymbol{N}_{\varepsilon} \mathrm{d} \Omega \\
\boldsymbol{G}=\int_{\Omega} \boldsymbol{N}_{\varepsilon}^{T} \mathbf{D}_{\mathbf{s}} \boldsymbol{B}_{\boldsymbol{u}} \mathrm{d} \Omega \\
\boldsymbol{K}=\int_{\Omega} \boldsymbol{B}_{u}^{T} \mathbf{D}_{\mathrm{s}} \boldsymbol{B}_{u} \mathrm{~d} \Omega \\
\boldsymbol{F}=\int_{\Omega} \boldsymbol{N}_{\boldsymbol{u}}^{T} \boldsymbol{f} \mathrm{d} \Omega+\int_{\Gamma_{t}} \boldsymbol{N}_{\boldsymbol{u}}^{T} \overline{\boldsymbol{t}} \mathrm{d} \Gamma
\end{gathered}
$$


where $\left[\begin{array}{ll}\boldsymbol{E} & \boldsymbol{U}\end{array}\right]^{T}$ is the array of nodal values of strains and displacements. $\boldsymbol{M}$ is a mass like projection matrix, $\boldsymbol{G}$ is the discrete gradient matrix, $\boldsymbol{K}$ is the standard stiffness matrix and $\boldsymbol{F}$ is the vector of external nodal forces.

In the system (40), the nodal values $\boldsymbol{E}$ can be formally eliminated to write the solution in terms of the nodal displacements $\boldsymbol{U}$ only, as follows. From the first equation in (40), the nodal values for the strains $\boldsymbol{E}$ can be obtained as

$$
\boldsymbol{E}=\boldsymbol{M}^{-1} \boldsymbol{G} \boldsymbol{U}
$$

which can be substituted into the second equation to yield

$$
U=\left(G_{\tau}^{T} M_{\tau}^{-1} G_{\tau}+K_{\tau}\right)^{-1} F
$$

This defines $\boldsymbol{K}^{\text {mix }}=\boldsymbol{G}_{\boldsymbol{\tau}}^{T} \boldsymbol{M}_{\boldsymbol{\tau}}^{-1} \boldsymbol{G}_{\boldsymbol{\tau}}+\boldsymbol{K}_{\boldsymbol{\tau}}$ as the stiffness matrix of the enhanced mixed FE formulation. Note that this definition is only formal, as $\boldsymbol{K}^{\text {mix }}$ cannot be assembled in an elementby-element fashion, nor it needs to be considered explicitly.

\section{Numerical simulations}

In this section, five sets of numerical simulations are performed using the isotropic and orthotropic damage models and the mixed FE formulation presented earlier. Numerical simulations are compared to theoretical or experimental solutions reported in the literature. The simulations are:

1. Willam's test

2. Traction tests on solid and hollow cylindrical specimens

3. Mixed mode bending tests

4. Mixed mode shearing-tension tests

5. Torsion test on a solid cylindrical specimen

Simulations 2 to 5 have been performed using 3D finite elements. No tracking technique is used in any of the cases. A stabilization parameter $\tau_{\varepsilon}=0.1$ is used in all the simulations.

Calculations are performed with an enhanced version of the finite element program COMET [33]. Pre- and post-processing are done with GiD [34], developed at CIMNE (International Center for Numerical Methods in Engineering).

\subsection{Willam's Test}

Willam's numerical test is used to highlight the difference between the isotropic and orthotropic damage models under shear loading. The test was proposed in reference [10] and it is regularly used to assess the performance of nonlinear damage constitutive laws [1, 31, 35-37].

The test examines the behavior of the isotropic and orthotropic damage models when the principal directions of strain rotate. The Rankine criterion is adopted in all cases. 
The test is performed with a single quadrilateral 2D element in plane stress conditions. The material properties are shown in Table 1. The loading is applied in two stages via increments of displacements. In the first stage, a uniaxial displacement is imposed in the horizontal X direction; as a consequence, deformation occurs in the $\mathrm{X}$ and $\mathrm{Y}$ directions, due to Poisson's effect. This leads to strains with an incremental ratio of $\Delta \varepsilon_{x x}: \Delta \varepsilon_{y y}: \Delta \gamma_{x y}=1:-v: 0$. The first stage ends when the stress $\sigma_{x x}$ reaches the value of the uniaxial tensile strength. In the second stage, displacements are imposed such that the incremental ratio of strains is $\Delta \varepsilon_{x x}: \Delta \varepsilon_{y y}: \Delta \gamma_{x y}=$ 1: 1.5: 1 until the complete failure of the material. Note that in the second stage of the test the principal directions of strain change, as the ratio $\varepsilon_{x x}: \varepsilon_{y y}: \gamma_{x y}$ changes at each step.

A constitutive model is said to pass Willam's test if (a) the predicted maximum principal stress does not exceed the uniaxial tensile strength and (b) all computed stress components tend to zero asymptotically $[35,37]$.

\begin{tabular}{|c|c|}
\hline Young's Modulus & $30 \cdot 10^{9} \mathrm{~Pa}$ \\
\hline Poisson's Ratio & 0.2 \\
\hline Tensile Strength & $3.0 \cdot 10^{6} \mathrm{~Pa}$ \\
\hline Fracture Energy & $200 \mathrm{~J} / \mathrm{m}^{2}$ \\
\hline
\end{tabular}

Table 1. Material parameters of Willam's test

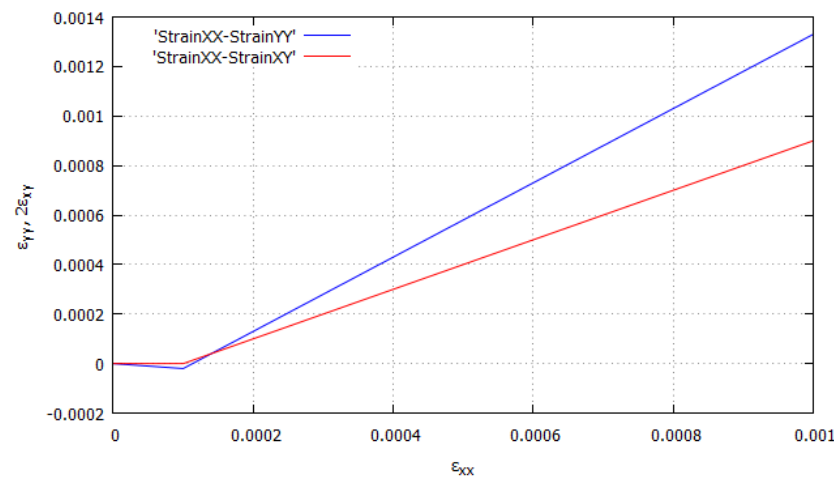

Figure 1. Imposed $\varepsilon_{x x}, \varepsilon_{y y}$ and $\varepsilon_{x y}$ during Willam's test

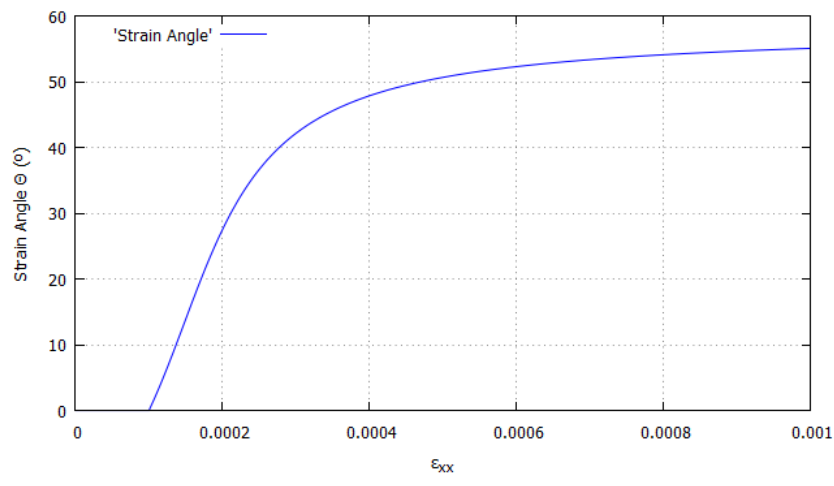

Figure 2. Evolution of the angle (degrees) of the maximum principal strain with respect to the $\mathrm{X}$ direction 


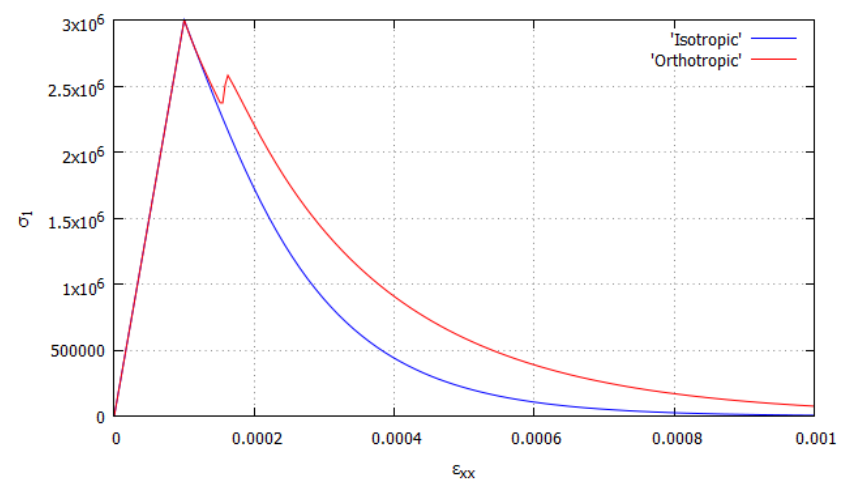

Figure 3. Computed maximum principal stress in Willam's test

\section{Isotropic vs orthotropic damage}

Figure 1 shows the evolution of strains during Willam's test. The two stages of the test can be easily identified. The rotation of the principal directions of strain that occurs during the process is depicted in Figure 2. In the first stage, the principal directions of strain are aligned with the axis of the element. In the second stage, strains rotate and the angle of the maximum principal strain with respect the $\mathrm{X}$ direction tends asymptotically to $58.28^{\circ}$, which corresponds to the limit case $\varepsilon_{x x}: \varepsilon_{y y}: \gamma_{x y}=1: 1.5: 1$

In Figure 3 the evolution of the maximum principal stress for the two constitutive laws is shown. In the isotropic case, the principal stress decreases asymptotically to zero after reaching the peak. In the orthotropic case, a second peak of stresses appears shortly after the occurrence of the first one. After that, the major principal stress also decreases asymptotically to zero.

In Figure 4, the evolution of the $\sigma_{x x}, \sigma_{y y}$, and $\tau_{x y}$ is depicted. For isotropic damage, there is a single damage index affecting the evolution of all the stresses components $\sigma_{x x}, \sigma_{y y}$, and $\tau_{x y}$. On the contrary, with orthotropic damage, components $\sigma_{x x}$ and $\sigma_{y y}$ behave in a much more independent way, as each principal direction of stress is affected by a different damage index.

Figure 4 also shows the rotation of principal directions of strain and stress. In the first stage, $\sigma_{y y}$ and $\tau_{x y}$ are zero and the maximum principal stress $\sigma_{1}$ is in the direction of $\sigma_{x x}$. In the second stage, the ratio between $\sigma_{x x}, \sigma_{y y}$, and $\tau_{x y}$ is constantly changing, making the principal directions of stresses $\sigma_{1}$ and $\sigma_{2}$ to continuously rotate.

Figure 5 shows the evolution of the angle of the maximum principal stress with respect the $\mathrm{X}$ direction for the isotropic and orthotropic cases. As it can be observed, the two results are overlapping, demonstrating that in the two damage models the strains and stresses are coaxial. It should be noted that the angle of the maximum principal stress tends asymptotically to the same angle as the one corresponding to strains in Figure 2.

The two models pass the Willam's test according to the aforementioned criteria. 

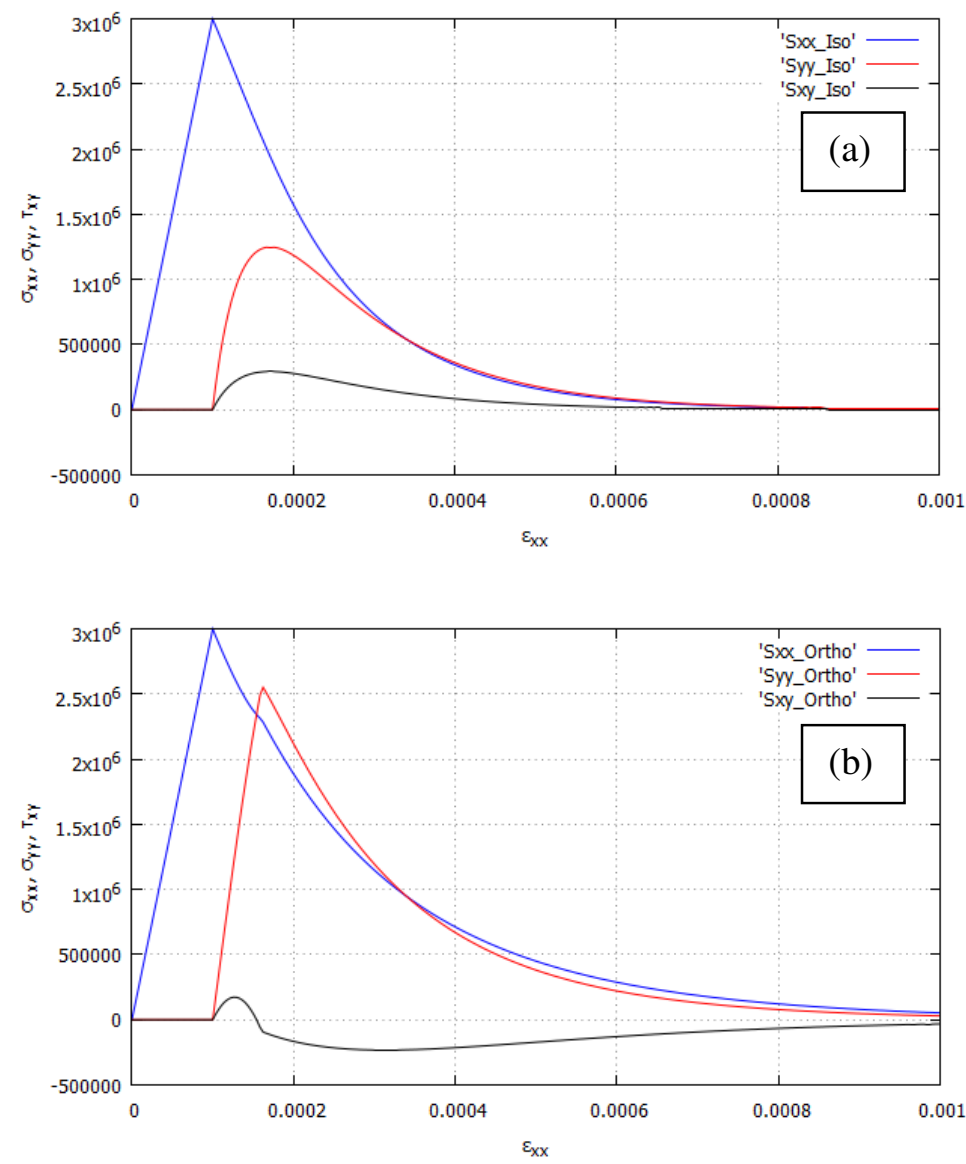

Figure 4. Evolution of $\sigma_{x x}, \sigma_{y y}$ and $\tau_{x y}$ stresses in Willam's test for the (a) isotropic and (b) orthotropic models

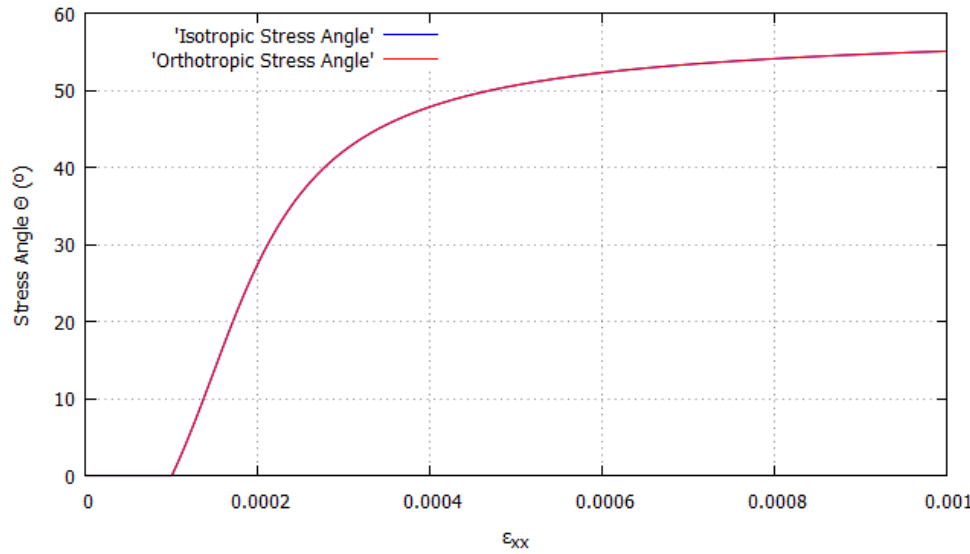

Figure 5. Evolution of the angle (degrees) of the maximum principal stress with respect to the $\mathrm{X}$ direction 


\subsection{Traction test on solid and hollow cylindrical specimens}

The numerical analysis of cylindrical concrete specimens subjected to axial straining is performed. The objective of this example is to test the behavior of several damage models with regard to their sensitivity to Poisson's ratio.

Computations are performed with the Beltrami and Modified Beltrami criteria. First, a comparison is performed between the isotropic versions of the models. Afterwards, the orthotropic Beltrami model is also employed.

Two cases are considered. In the first one, the cylinder is solid, and in the second one, the cylinder is hollow. The cylinder has a $50 \mathrm{~mm}$ radius and is $450 \mathrm{~mm}$ long in the solid case and $500 \mathrm{~mm}$ long in the hollow case. The thickness of the hollow specimen is $5 \mathrm{~mm}$. A small hole of $5 \mathrm{~mm}$ radius has been introduced in the middle of the specimens to fix the occurrence of fracture. The material properties are shown in Table 2. Simulations are performed with different Poisson's ratio values. Opposite increments of vertical displacement are applied at the top and bottom surfaces of the specimen.

\begin{tabular}{|c|c|c|}
\hline Case & Solid & Hollow \\
\hline Young's Modulus & $38 \cdot 10^{9} \mathrm{~Pa}$ & $38 \cdot 10^{9} \mathrm{~Pa}$ \\
\hline Tensile Strength & $2.3 \cdot 10^{6} \mathrm{~Pa}$ & $3.0 \cdot 10^{6} \mathrm{~Pa}$ \\
\hline Fracture Energy & $80 \mathrm{~J} / \mathrm{m}^{2}$ & $80 \mathrm{~J} / \mathrm{m}^{2}$ \\
\hline
\end{tabular}

Table 2. Material parameters of the traction test
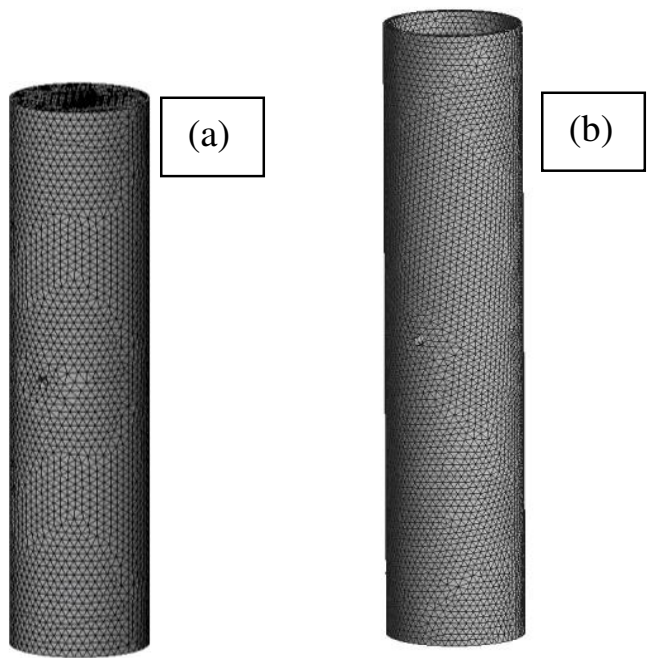

Figure 6. Meshes used for the analyses of the traction test for the (a) solid and (b) hollow specimens

In both cases, the specimens are discretized with fully unstructured meshes of tetrahedral elements, shown in Figure 6 . For the solid specimen the elements have a size of $6 \mathrm{~mm}$, resulting in a mesh of 21,986 nodes; in the hollow specimen, the elements are of $5 \mathrm{~mm}$, ensuing a mesh of 15,823 nodes. 


\section{Influence of Poisson's ratio}

Figure 7 shows the computed crack surfaces in the solid specimen obtained with the Beltrami criterion for different values of Poisson's ratio, plotted as the isolevel surface of the norm of vertical displacements. Corresponding results, also with the Beltrami criterion, are shown in Figure 8 for the hollow specimen.

It can be seen that different values of Poisson's ratio produce crack surfaces at different angles with the horizontal plane (orthogonal to the axial stress). This angle has been theoretically computed for Beltrami's criterion in reference [28] depending on the value of Poisson's ratio for plane stress and plane strain behavior. The solid specimen behaves closely to plane strain conditions while the hollow case behaves similarly to plane stress conditions. Table 3 compares the expected theoretical angles and the computed ones for each case. It can be seen that the computed results are very close to the expected theoretical solutions.

Figure 9 shows the force-displacement curves for both the solid and the hollow specimens computed with the Beltrami criterion for several Poisson's ratio values. It can be seen that the results in terms of dissipated energy depend on Poisson's ratio. Note that the load capacity of the specimens differs very little from the values corresponding to perfectly brittle failure $(18.06 \mathrm{kN}$ and $4.48 \mathrm{kN}$ for the solid and hollow specimens, respectively) and are quite independent of Poisson's ratio. Contrariwise, the dissipated energy increases with the Poisson's ratio, as the failure mechanism and the corresponding crack surface varies.

The results computed with the Modified Beltrami criterion in Figure 10 are nearly identical for all values of Poisson's ratio, showing a horizontal crack surface. This result was also theoretically derived in reference [28]. It is to be expected as the influence of Poisson's ratio in the formulation of the modified model is very much reduced (see equations (15) and (16)).

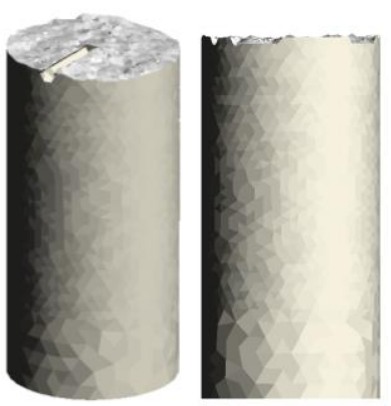

Poisson's ratio: 0.00

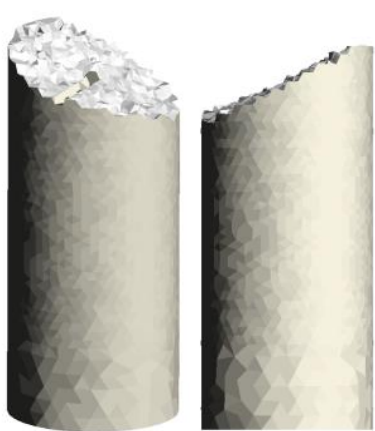

Poisson's ratio: 0.30

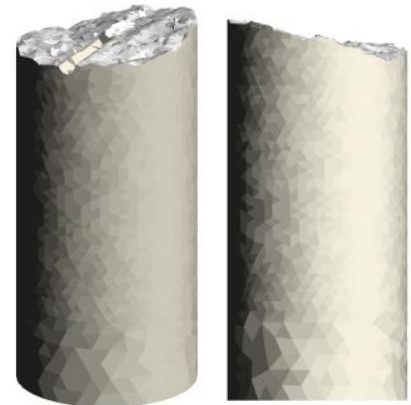

Poisson's ratio: 0.15

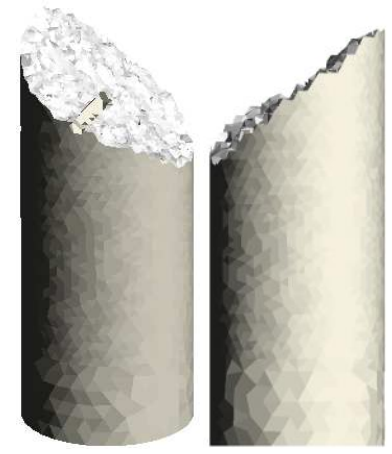

Poisson's ratio: 0.45

Figure 7. Crack surfaces of the traction test, solid specimen, with the Beltrami criterion for several Poisson's ratio values 


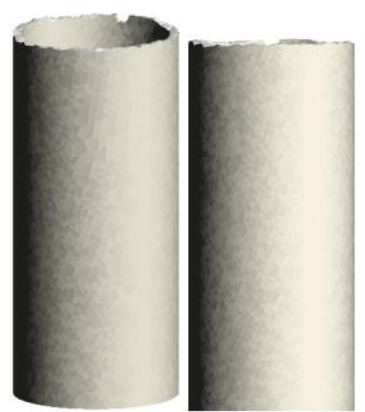

Poisson's ratio: 0.00

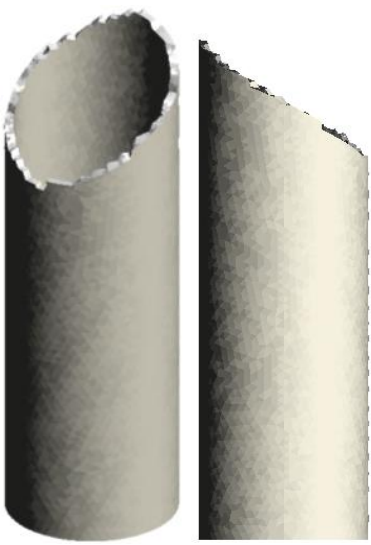

Poisson's ratio: 0.30

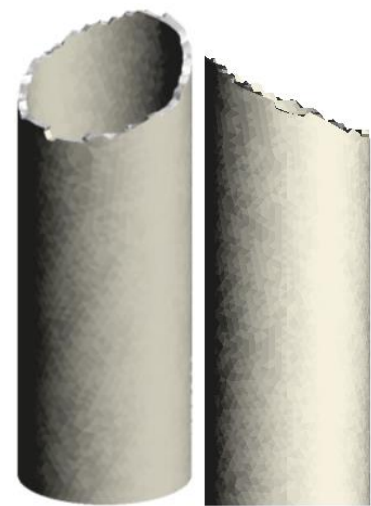

Poisson's ratio: 0.15

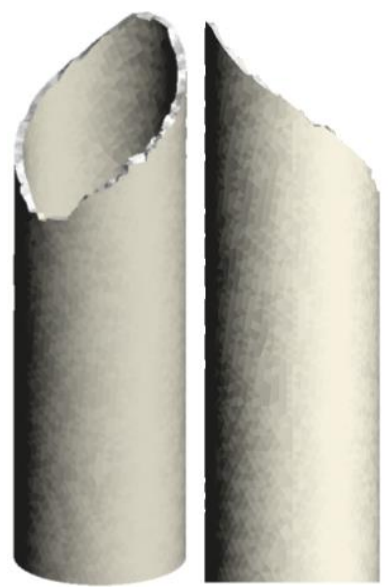

Poisson's ratio: 0.45

Figure 8. Crack surfaces of the traction test, hollow specimen, with the Beltrami criterion for several Poisson's ratio values

\begin{tabular}{|c|c|c|c|c|c|c|}
\hline Poisson's ratio & $\begin{array}{c}\text { Theoretical Angle } \\
\text { Plane Strain }\end{array}$ & $\begin{array}{c}\text { Computed Angle } \\
\text { Solid Specimen }\end{array}$ & Error (\%) & $\begin{array}{c}\text { Theoretical Angle } \\
\text { Plane Stress }\end{array}$ & $\begin{array}{c}\text { Computed Angle } \\
\text { Hollow Specimen }\end{array}$ & Error (\%) \\
\hline 0.00 & $0.00^{\circ}$ & $0^{\circ}$ & 0.00 & $0.00^{\circ}$ & $0^{\circ}$ & 0.00 \\
\hline 0.15 & $21.17^{\circ}$ & $19^{\circ}$ & 10.25 & $22.79^{\circ}$ & $23^{\circ}$ & 0.92 \\
\hline 0.30 & $28.71^{\circ}$ & $29^{\circ}$ & 1.01 & $33.21^{\circ}$ & $33^{\circ}$ & 0.63 \\
\hline 0.45 & $33.85^{\circ}$ & $34^{\circ}$ & 0.44 & $42.13^{\circ}$ & $40^{\circ}$ & 5.03 \\
\hline
\end{tabular}

Table 3. Theoretical and computed angles of the crack surfaces in the traction test 

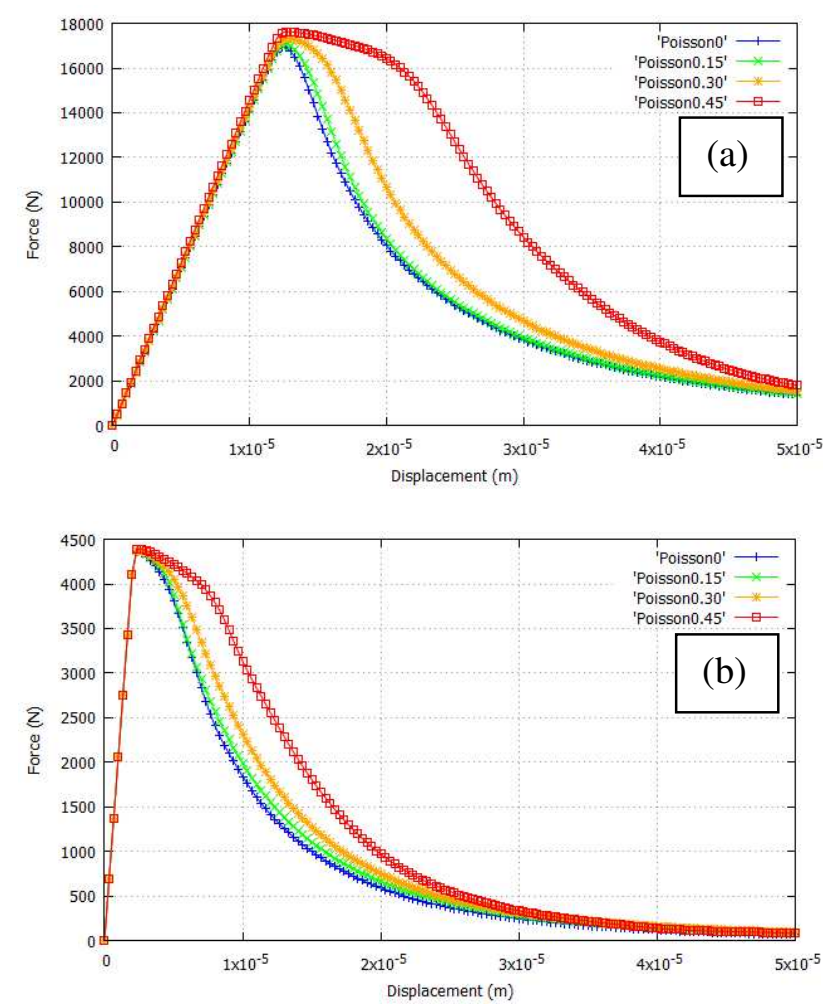

Figure 9. Force-displacement curves of the traction test computed with the Beltrami criterion for (a) the solid specimen and (b) the hollow specimen
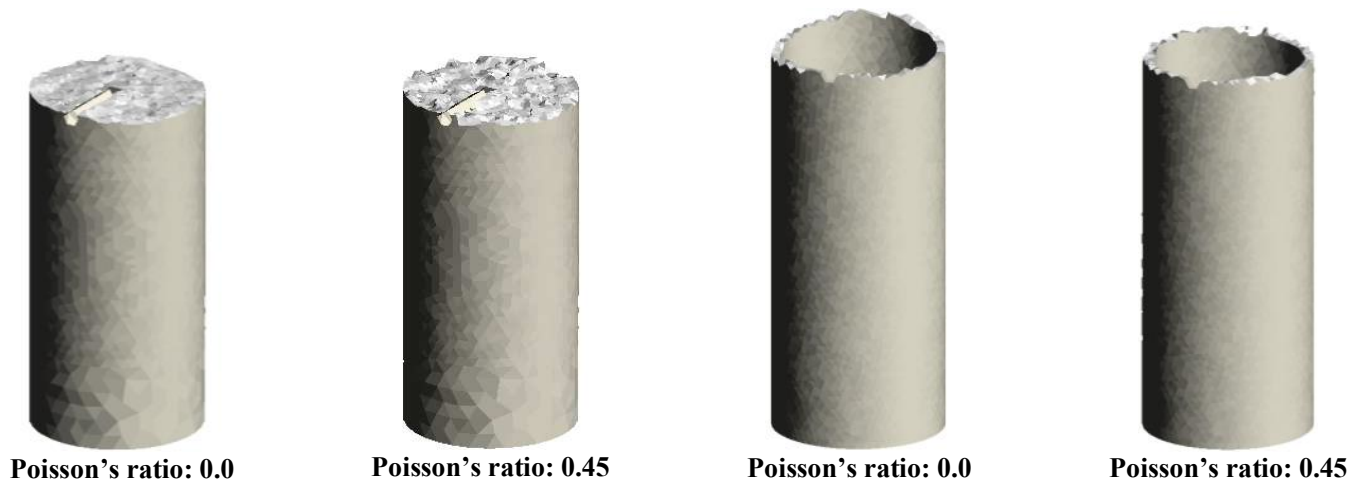

Figure 10. Crack surfaces of the traction test, with the Modified Beltrami criterion

\section{Isotropic vs orthotropic models}

In this section, the results obtained with the isotropic and orthotropic Beltrami models are compared. As it can be seen in the computed crack surfaces of Figures 11 and 12, and in Table 4, the crack surface angles are different. Compared to the isotropic results of Figures 7 and 8, the orthotropic damage crack surfaces have an angle which is systematically and significantly smaller for the same Poisson's ratio value. Although no theoretical confirmation is available, this difference is attributed to the reduction of the effective Poisson's effect in orthotropic models. 

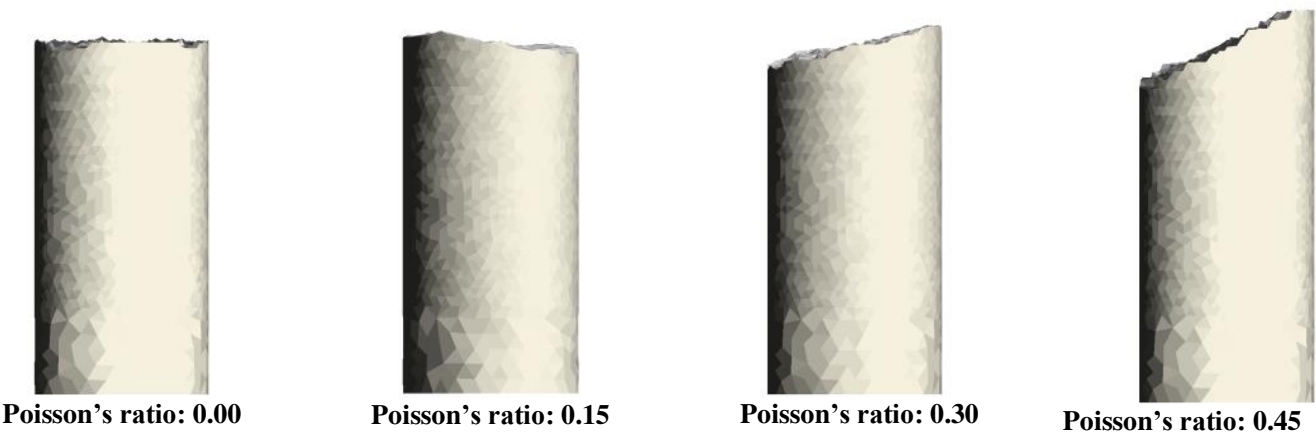

Figure 11. Crack surfaces of the traction test, solid specimen, computed with the orthotropic Beltrami criterion
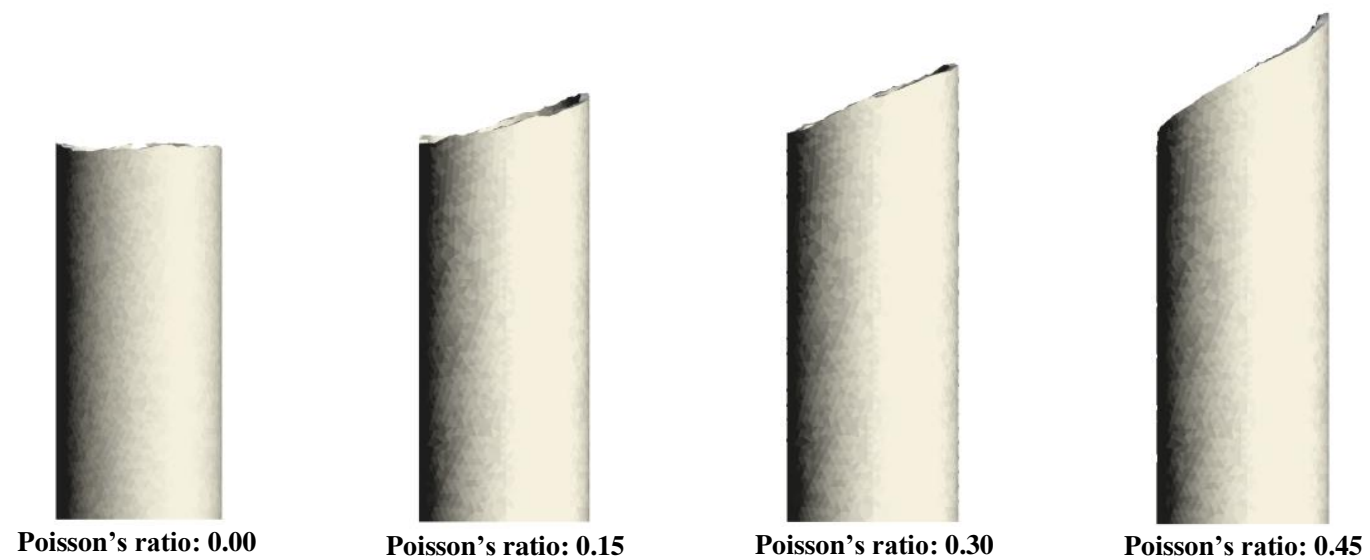

Figure 12. Crack surfaces of the traction test, hollow specimen, computed with the orthotropic Beltrami criterion

\begin{tabular}{|c|c|c|c|c|}
\hline Poisson's ratio & $\begin{array}{c}\text { Isotropic Beltrami } \\
\text { Solid Specimen }\end{array}$ & $\begin{array}{c}\text { Orthotropic Beltrami } \\
\text { Solid Specimen }\end{array}$ & $\begin{array}{c}\text { Isotropic Beltrami } \\
\text { Hollow Specimen }\end{array}$ & $\begin{array}{c}\text { Orthotropic Beltrami } \\
\text { Hollow Specimen }\end{array}$ \\
\hline 0.00 & $0^{\circ}$ & $0^{\circ}$ & $0^{\circ}$ & $0^{\circ}$ \\
\hline 0.15 & $19^{\circ}$ & $10^{\circ}$ & $23^{\circ}$ & $16^{\circ}$ \\
\hline 0.30 & $29^{\circ}$ & $14^{\circ}$ & $33^{\circ}$ & $22^{\circ}$ \\
\hline 0.45 & $34^{\circ}$ & $26^{\circ}$ & $40^{\circ}$ & $29 \circ$ \\
\hline
\end{tabular}

Table 4. Computed angles of the crack surfaces for the isotropic and orthotropic Beltrami damage models in the traction test

\subsection{Mixed mode bending test}

In this section, a notched beam subjected to a mixed mode bending test is considered. The experimental tests were first carried out by Arrea and Ingraffea [38] and then repeated by Gálvez and Cendón [39]. Reference [39] also performed numerical simulations with a cohesive interface method. Other numerical results are reported in [40-43]. Reference [40] considers a localization limiter to regularize the problem. In [41] an adaptive particle meshless method was used, while in [42] the boundary element method was employed. In [43] an interface finite element approach was adopted. 
This example is intended to assess the performance of the Rankine and Drucker-Prager criteria in mixed mode cracking situations. For comparison purposes, three different sets of beams with various geometries and properties were tested in [39]. The geometry of the three sets of beams is shown in Figure 13. In the A set, the beam thickness is $0.102 \mathrm{~m}$ and the notch $a$ is $0.070 \mathrm{~m}$ long. In the $\mathrm{B}$ and $\mathrm{C}$ sets, the beam is $0.152 \mathrm{~m}$ thick and the notch $a$ is $0.0824 \mathrm{~m}$ long. The different properties of the sets are shown in Table 5.

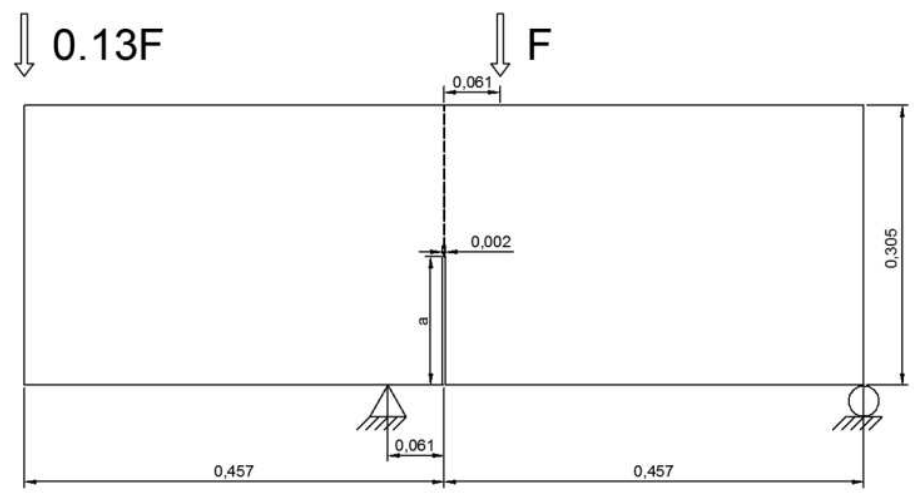

Figure 13. Geometry of the mixed mode bending test $(\mathrm{m})$

\begin{tabular}{|c|c|c|c|}
\hline Set & A & B & C \\
\hline Young's Modulus & $23.4 \cdot 10^{9} \mathrm{~Pa}$ & $24.8 \cdot 10^{9} \mathrm{~Pa}$ & $24.8 \cdot 10^{9} \mathrm{~Pa}$ \\
\hline Poisson's Ratio & 0.21 & 0.18 & 0.18 \\
\hline Tensile Strength & $4.6 \cdot 10^{6} \mathrm{~Pa}$ & $4.0 \cdot 10^{6} \mathrm{~Pa}$ & $3.7 \cdot 10^{6} \mathrm{~Pa}$ \\
\hline Fracture Energy & $75 \mathrm{~J} / \mathrm{m}^{2}$ & $125 \mathrm{~J} / \mathrm{m}^{2}$ & $130 \mathrm{~J} / \mathrm{m}^{2}$ \\
\hline Compressive Strength & $60.7 \cdot 10^{6} \mathrm{~Pa}$ & $45.5 \cdot 10^{6} \mathrm{~Pa}$ & $43.4 \cdot 10^{6} \mathrm{~Pa}$ \\
\hline
\end{tabular}

Table 5. Material properties of the mixed mode bending test

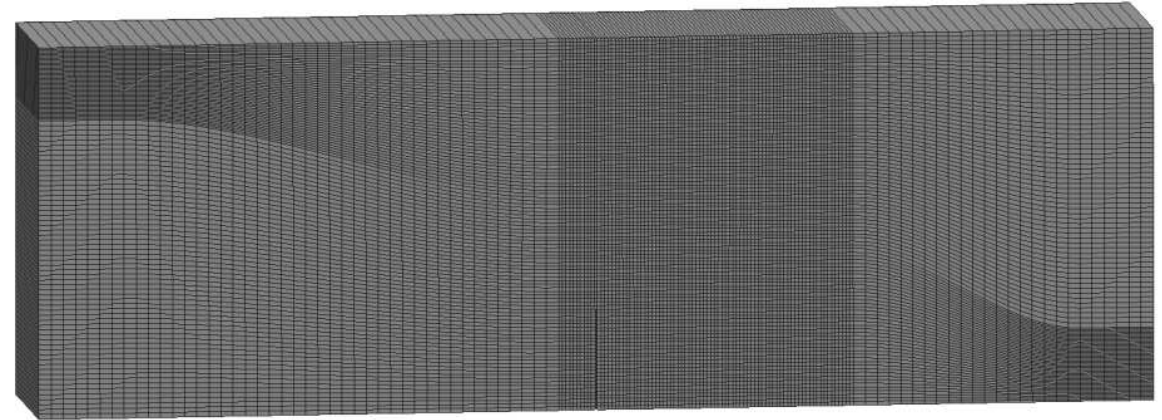

Figure 14. FE mesh used for the mixed mode bending test

The problem is solved using an arc-length algorithm controlling the crack mouth sliding displacement (CMSD) at the notch. For this example, 3D hexahedral elements are used, resulting in a fully structured mesh of 31,634 nodes and elements of $3 \mathrm{~mm}$, shown in Figure 14.

First, a comparison between the isotropic and orthotropic Rankine models is performed to assess their ability to reproduce mixed mode I and II failure. Then, a comparison is made between the isotropic and orthotropic Drucker-Prager models. Finally, the relative performance of the mixed and standard FE formulations is addressed. 


\section{Rankine isotropic vs orthotropic models}

Figure 15 shows damage contour fills for the sets A and C computed with the Rankine criterion. It can be seen that the results for the isotropic and orthotropic models are quite similar.

Crack trajectories are compared in detail in Figure 16 for all three sets. There, it can be seen that, for the A set, the computed crack paths with the different models are all very similar. In addition, they are all inside the experimental range of reference [39]. For the B and C sets, the results of the Rankine isotropic and orthotropic models are also very close, but none of them give results inside the experimental range.

The crack surface in the 3D analyses is depicted in Figure 17, plotted as an iso-level surface of the X-displacements. There, the crack surfaces of the different criteria considered for modeling set $\mathrm{C}$ can be observed.

In Figure 18, the force-CMSD curves are shown for each set. It can be seen that the isotropic and orthotropic models are very close and correlate very closely with the experiments.
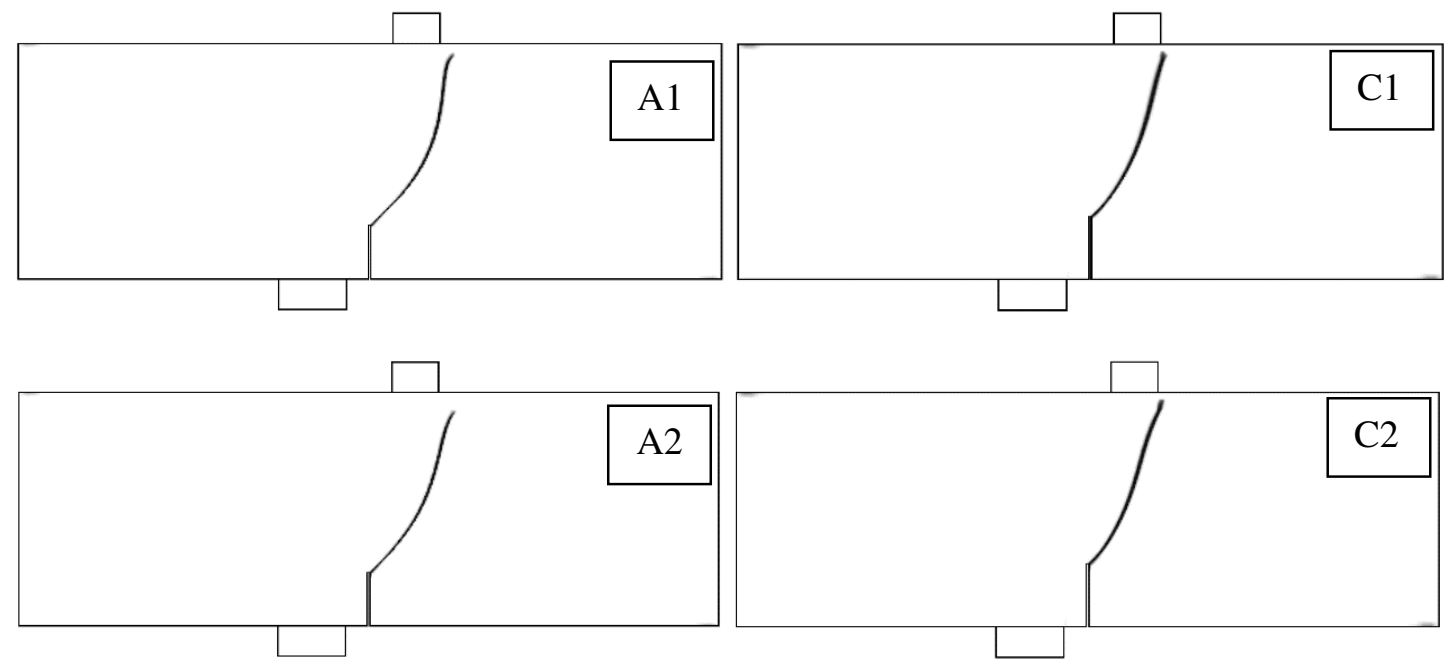

Figure 15. Damage contour fills of the mixed mode bending test, sets A and C, for the Rankine criterion with (1) isotropic damage and (2) orthotropic damage
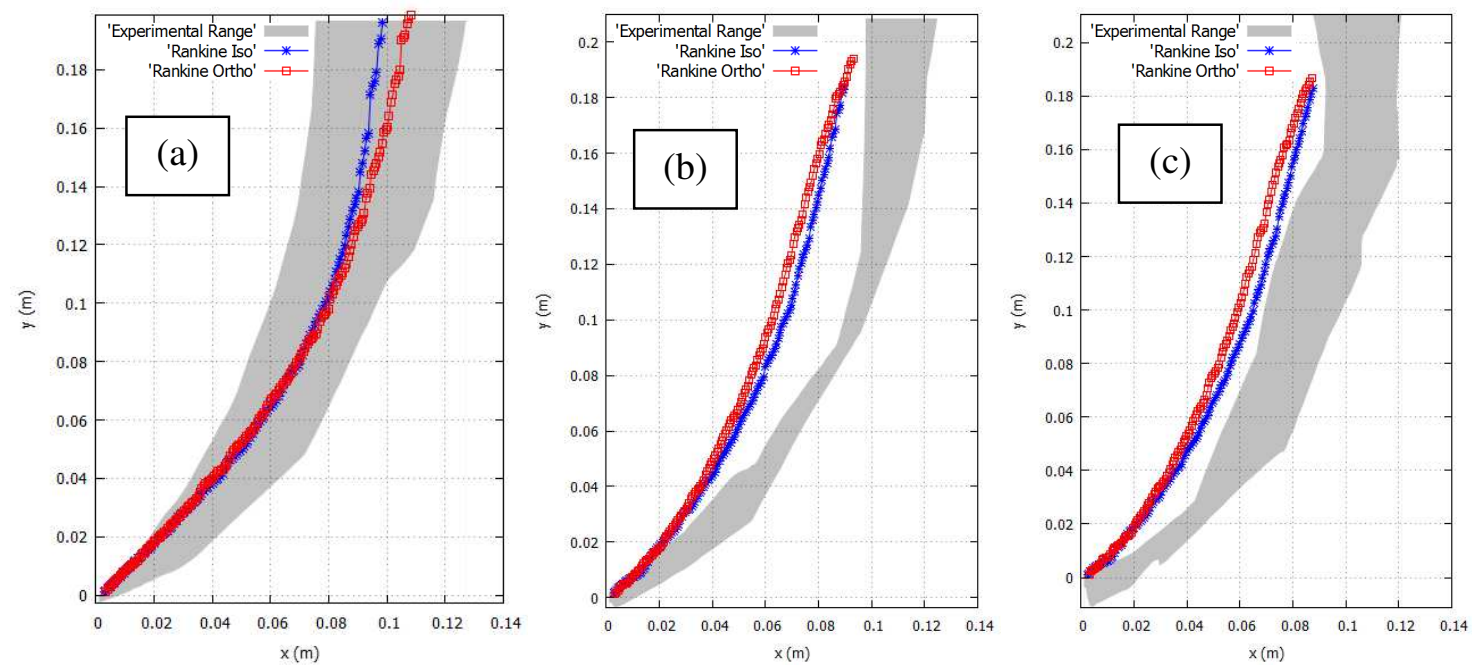

Figure 16. Crack paths compared to experimental results of the mixed mode bending test for the Rankine criterion (a) set $A$, (b) set $B$ and (c) set $C$. 

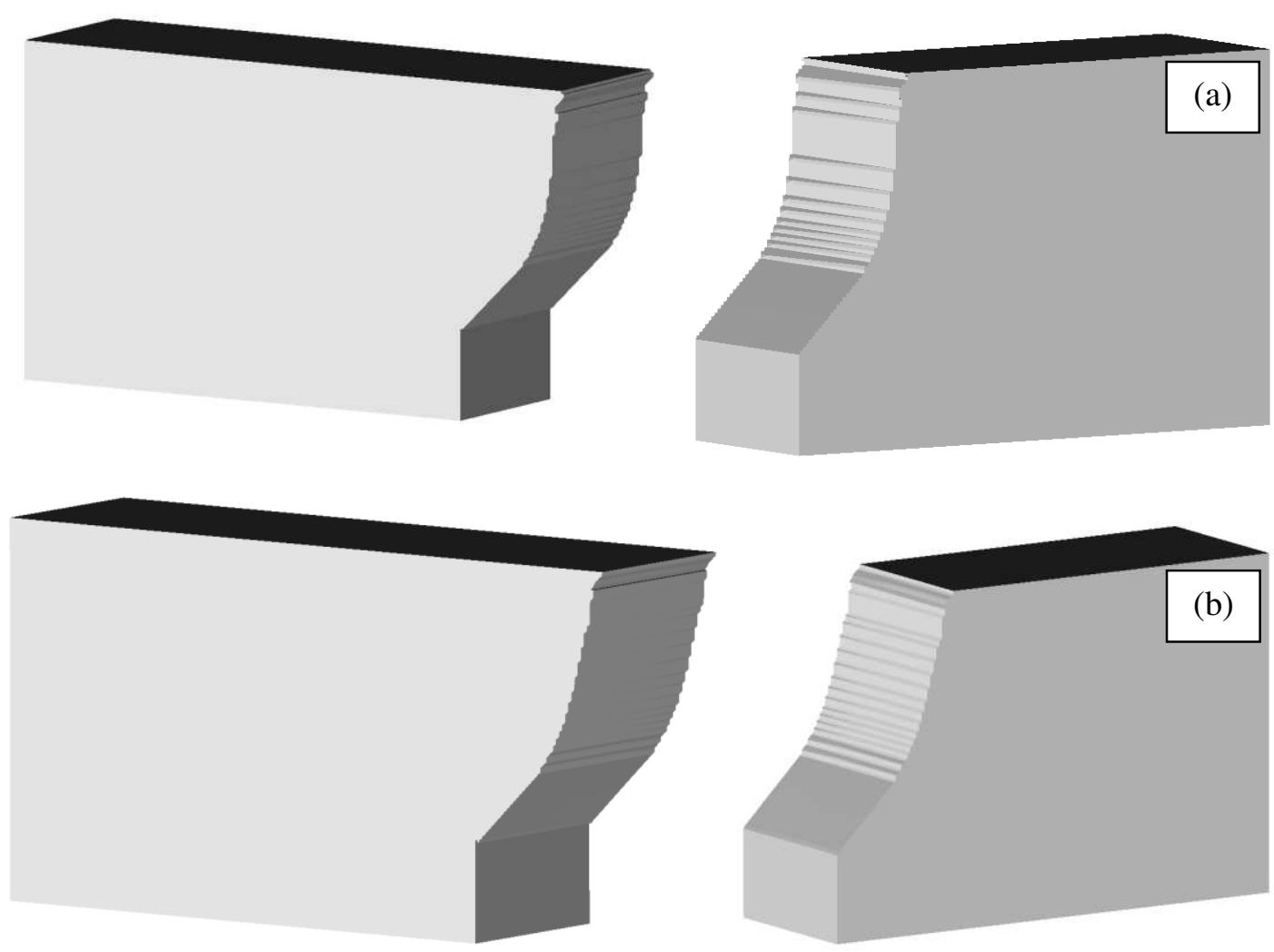

Figure 17. Crack surfaces of the mixed mode bending test, set $\mathrm{C}$, for the Rankine criterion with (a) isotropic and (b) orthotropic damage 

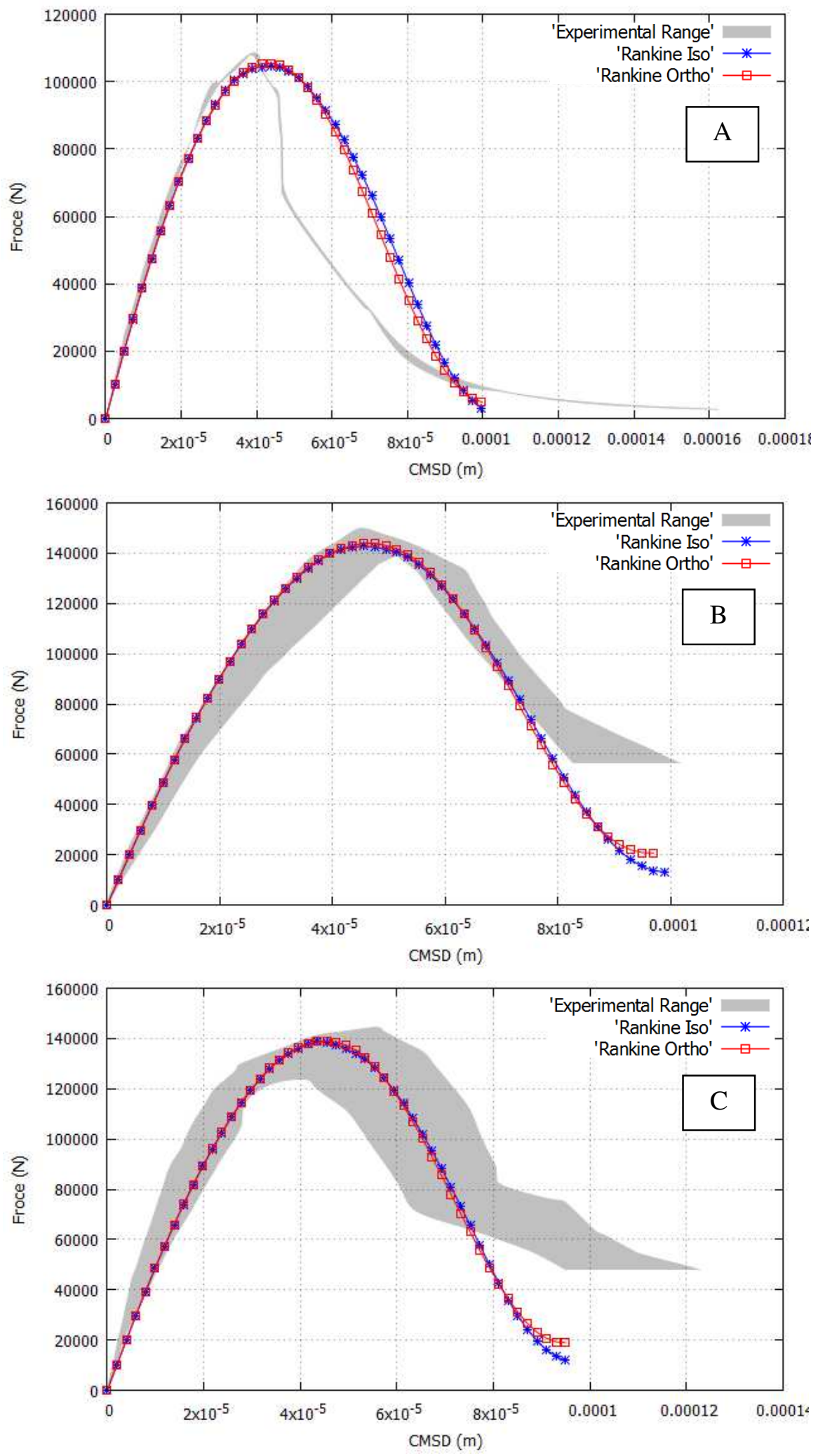

Figure 18. Force-CMSD curves of the mixed mode bending test for Rankine's model and sets A, B and $\mathrm{C}$ 


\section{Rankine vs Drucker-Prager damage criteria}

In the following, the use of the Drucker-Prager damage criterion has been considered. The corresponding isotropic and orthotropic models are used, as detailed in Section 2. In the orthotropic Drucker-Prager laws, see equation (28), damage only evolves in the directions where the corresponding principal stress is positive.
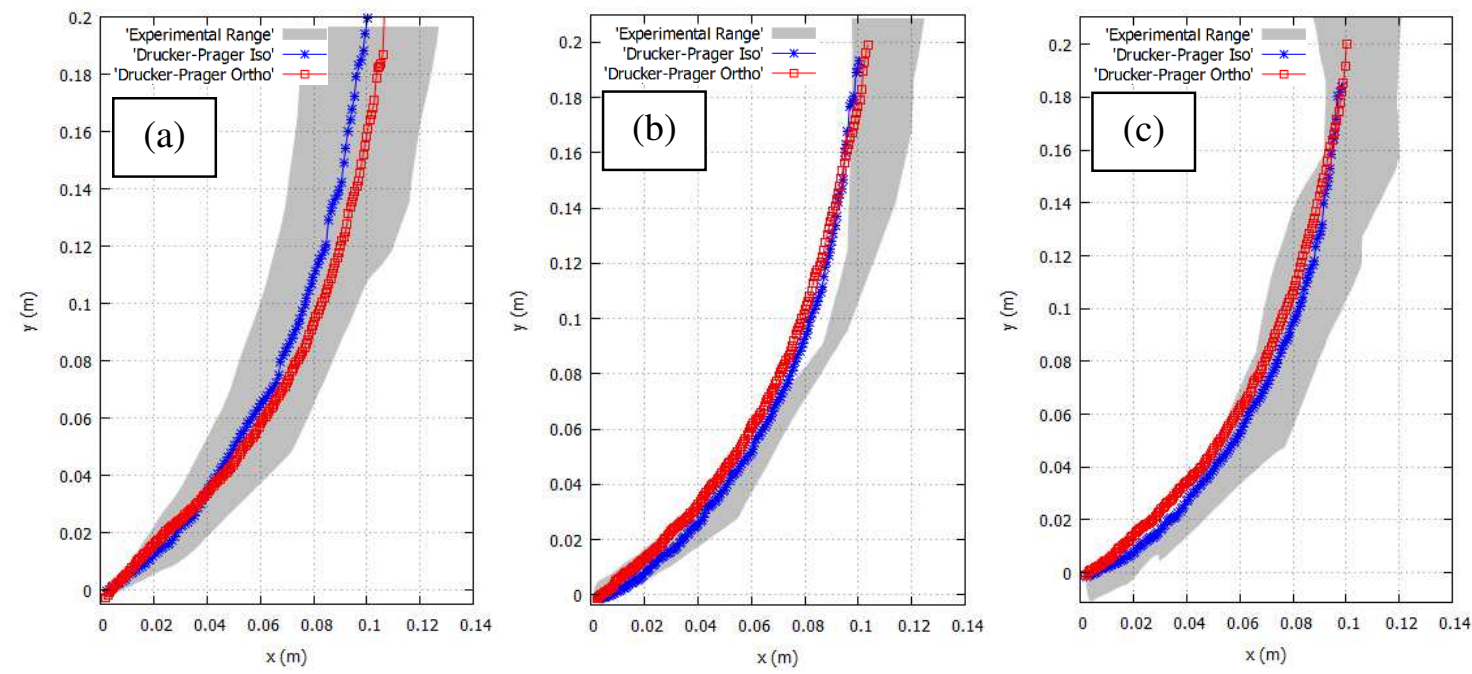

Figure 19. Crack paths compared to experimental results of the mixed mode bending test for the Drucker-Prager criterion (a) set A, (b) set B and (c) set C.

The crack paths of all three sets are included in Figure 19 for the isotropic and orthotropic Drucker-Prager models. It can be seen how isotropic and orthotropic models produce very similar results in terms of crack path. The crack surfaces are inside the experimental ranges for all three sets A, B, and C. Similar results were reported in the numerical simulations performed by [39], where a cohesive interface method was used.

In Figure 20 the computed crack surfaces of all three cases of unit $\mathrm{C}$ can be observed. The results captured with the Drucker-Prager orthotropic constitutive laws are very similar to the corresponding isotropic damage. Computed results show good agreement with the experimental surface reported in [38]. The Drucker-Prager criterion is also considered to perform better than Rankine's for mixed mode fracture in references [25, 32].

Figure 21 shows the force-CMSD curves of the three sets computed with the isotropic and orthotropic Drucker-Prager criteria. All the computed results are almost overlapping and in good agreement with the experiments.

In Figure 22 the evolution of the maximum principal stresses is depicted as the crack propagates in set A for the isotropic Rankine and Drucker-Prager models. Stresses concentrate at the tip of the crack making it to progress, following the path shown in Figure 19. Different damage criterions introduce different equivalent stresses and, therefore, they produce different crack paths. The Rankine and Drucker-Prager criterions produce similar but not identical crack trajectories, the crack corresponding to the latter being more curved. This is a direct consequence of the (slight) difference between the two different criteria for mixed stress states. The elastic principal stresses near the crack tip can be observed in Figure 23. 

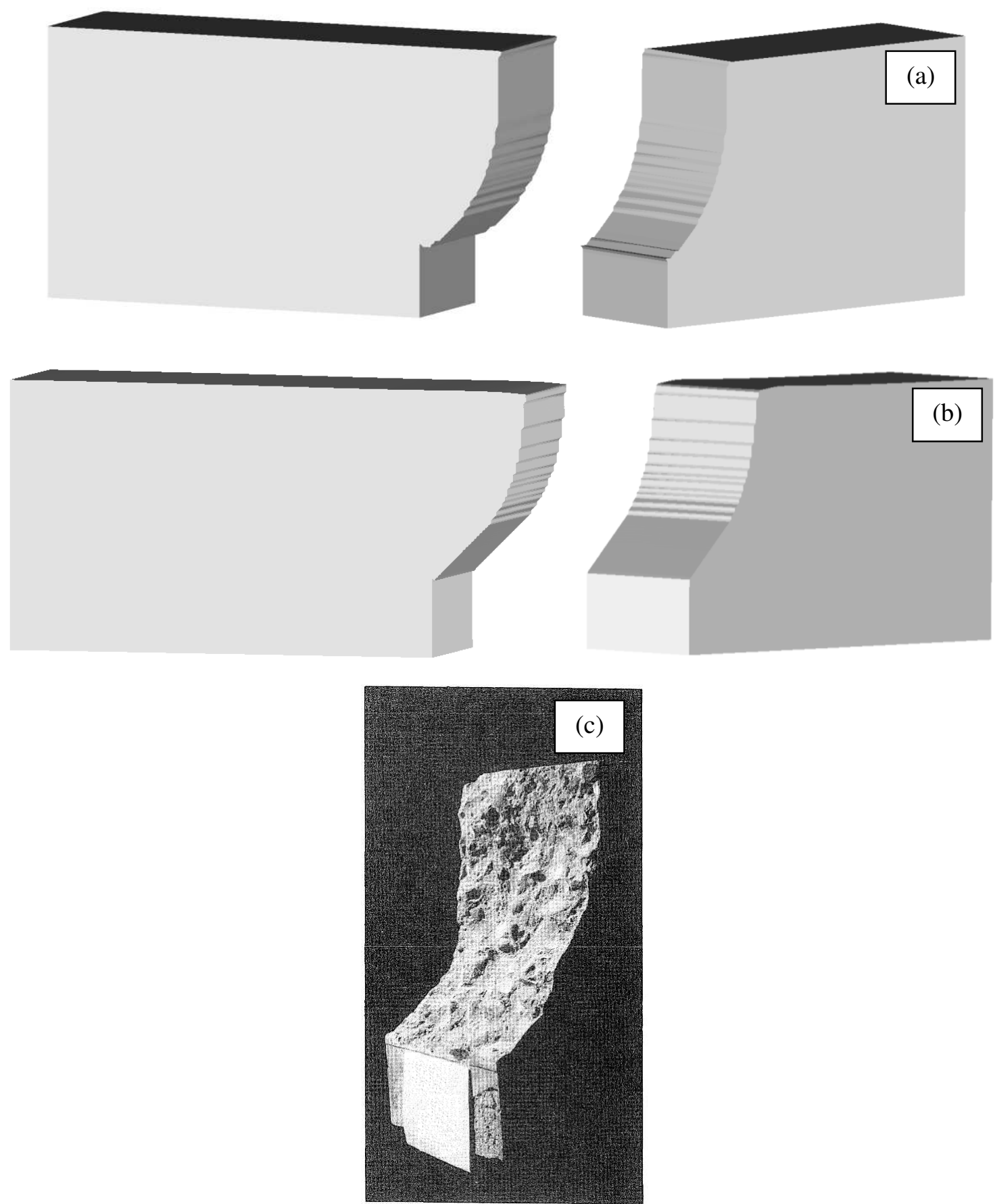

Figure 20. Crack surfaces, mixed mode bending test, set $\mathrm{C}$, for the Drucker-Prager criterion with (a) isotropic (b) orthotropic damage and (c) a photo of the crack surface in the experiment of [38] 

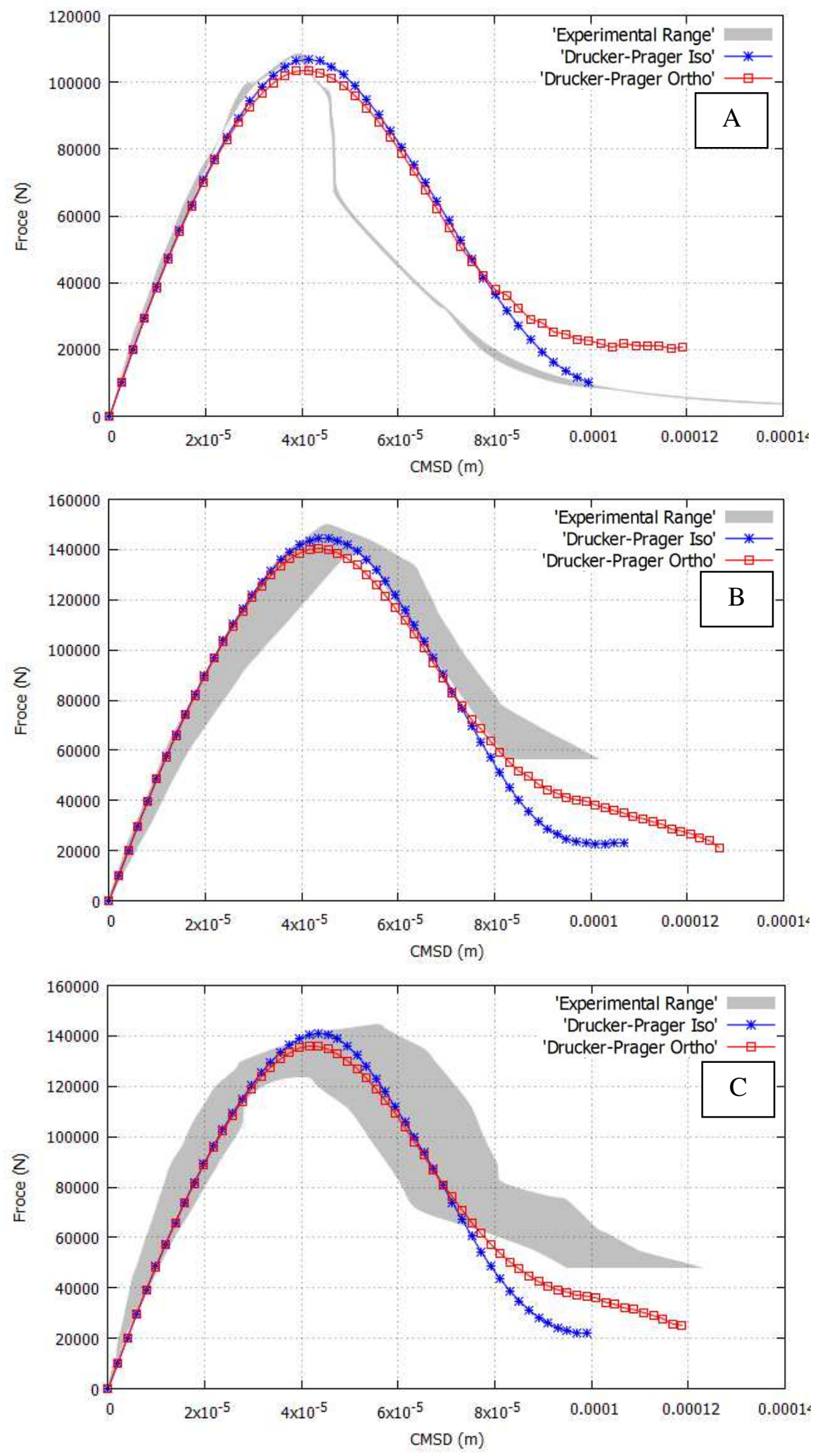

Figure 21. Force-CMSD curve of the mixed mode bending test for the Drucker-Prager model, sets $\mathrm{A}, \mathrm{B}$ and $\mathrm{C}$ 

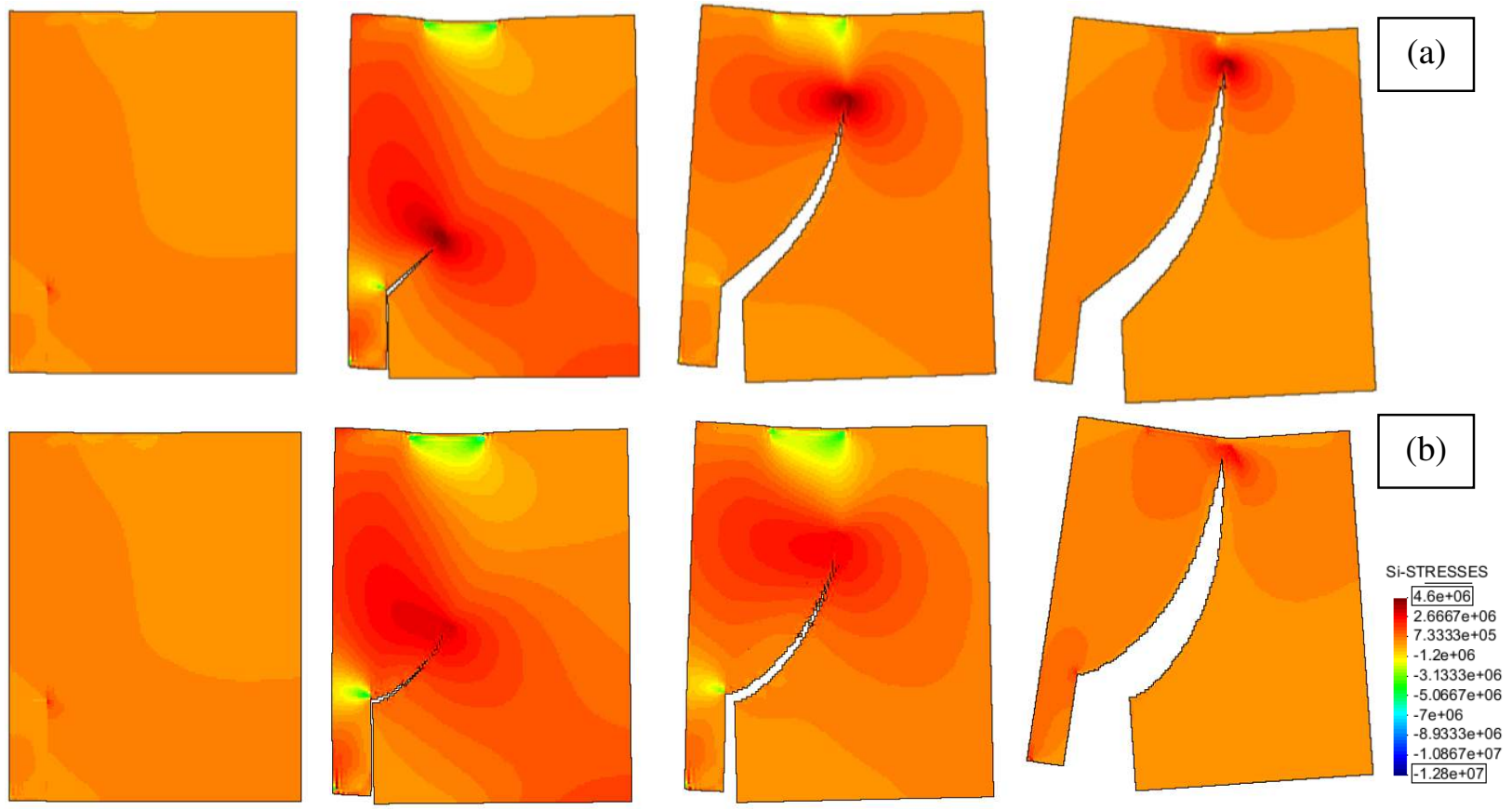

Figure 22. Stress evolution and crack propagation, in the set A, for the isotropic (a) Rankine and (b) Drucker-Prager models

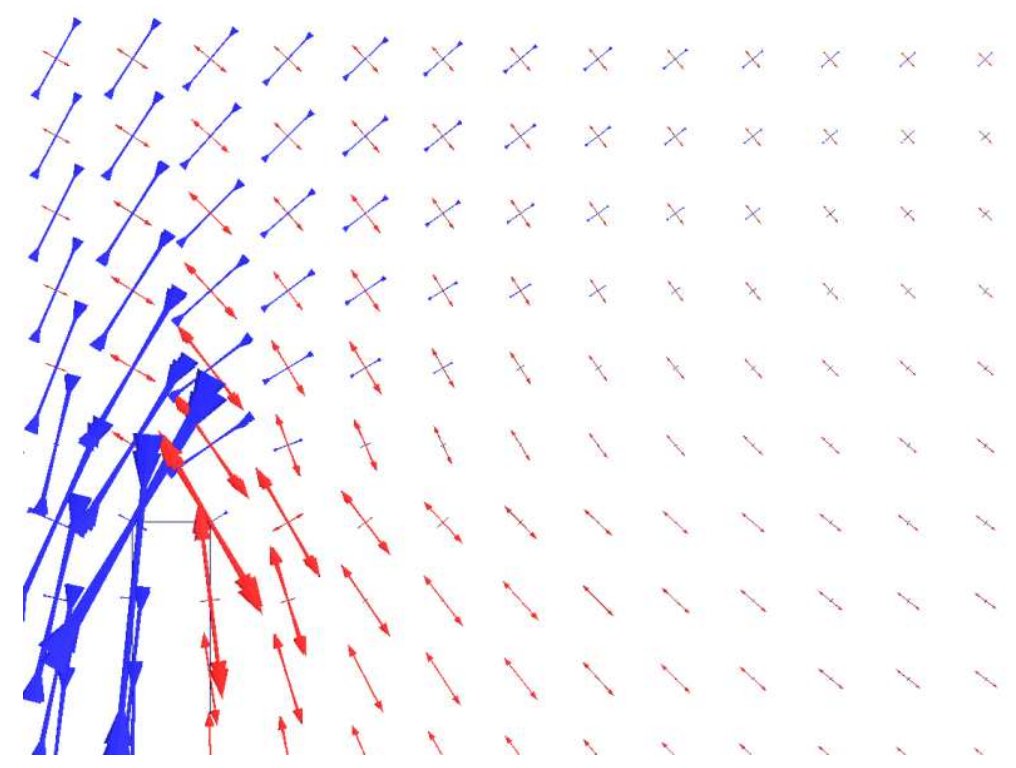

Figure 23. Elastic principal stresses near the crack tip

\section{Comparison with standard FE}

In this bending problem, isotropic and orthotropic models performed very similarly both for the Rankine and Drucker-Prager criteria. In this section, results for set $\mathrm{C}$ computed with standard finite elements are reported to show the relative improvement of the mixed formulation. 

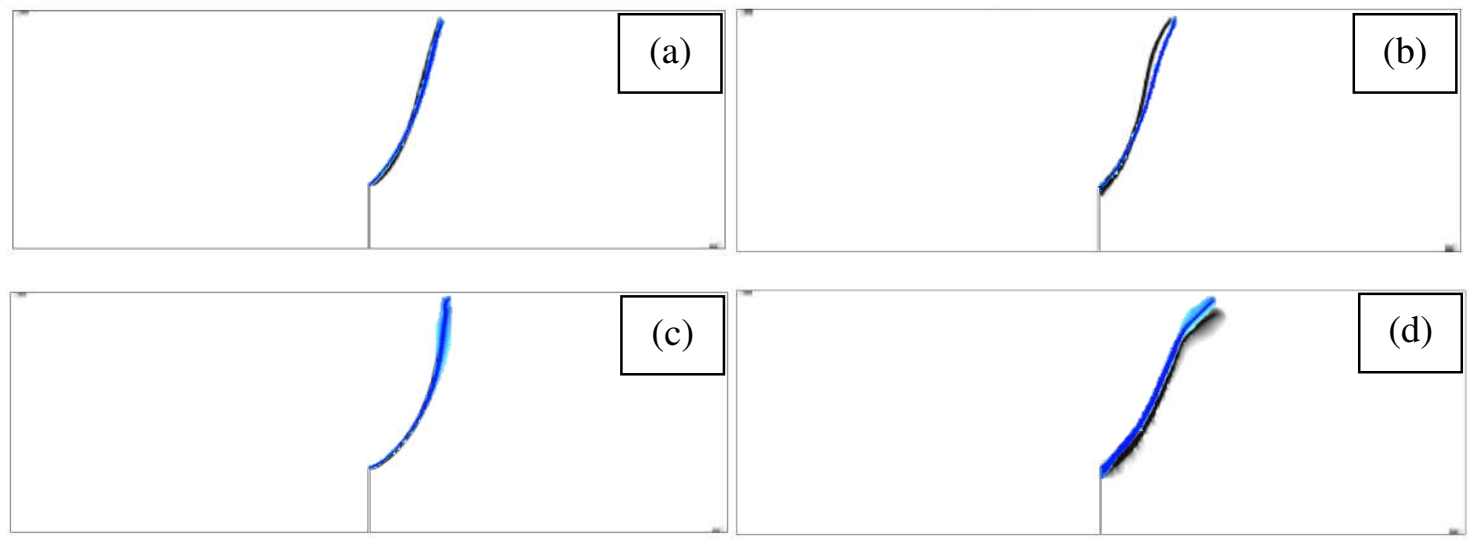

Figure 24. Damage contour fills of the mixed mode bending test, set C, computed with standard FE (black crack path) vs mixed FE (blue crack path) with (a) isotropic Rankine, (b) orthotropic Rankine, (c) isotropic Drucker-Prager and (d) orthotropic Drucker-Prager models
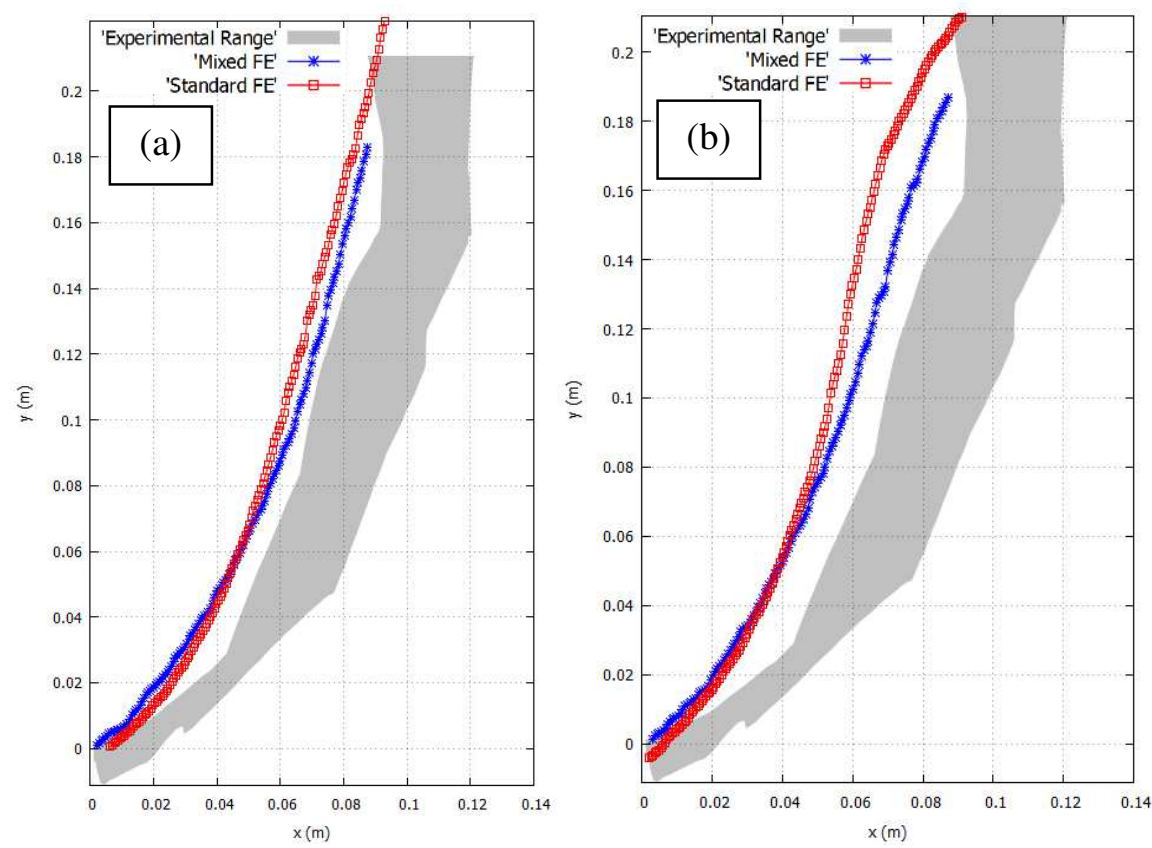

Figure 25. Crack paths compared to experimental results of the mixed mode bending test, set $\mathrm{C}$, for (a) isotropic and (b) orthotropic Rankine models

In Figure 24 the damage contour fills computed with standard FE, for the Rankine and DruckerPrager criteria, using isotropic and orthotropic damage, are compared to the results obtained with mixed FE. These are to be compared to Figure 15, where the corresponding results for the mixed formulation are given.

Details of the computed crack paths are given in Figures 25 and 26. It can be seen how the Rankine criterion also yields unsuitable results, outside the experimental range with standard FE. The mixed FE crack paths produce results slightly closer to the experiments. The Drucker-Prager model, on the other hand, results in crack paths which are also inside the experimental range when using standard finite elements. When using isotropic damage, the computed crack paths are very close, while when employing orthotropic models, they are different, especially in the last stages of the simulation. 

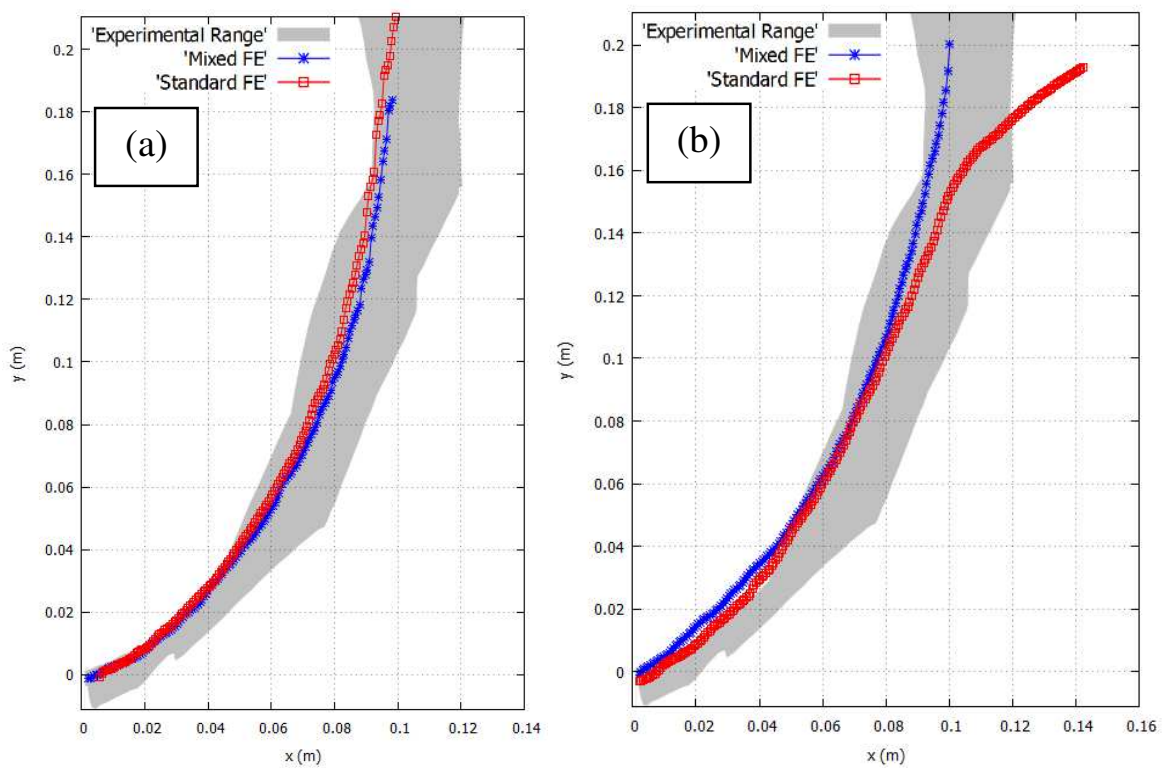

Figure 26. Crack paths compared to experimental results of the mixed mode bending test, set $\mathrm{C}$, for (a) isotropic and (b) orthotropic Drucker-Prager models

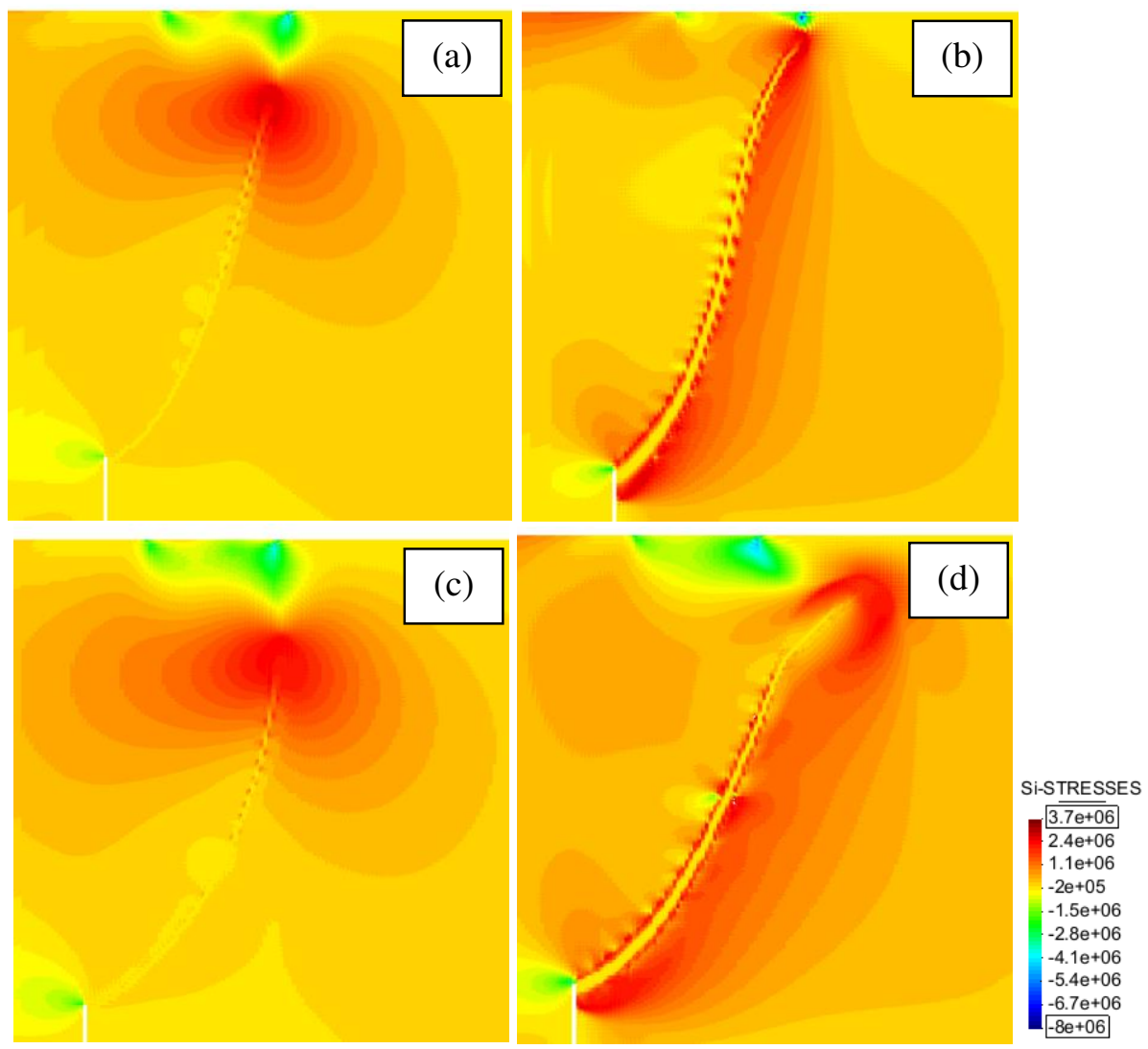

Figure 27. Maximum principal stress of the mixed mode bending test, set $C$, computed with standard FE with (a) isotropic Rankine, (b) orthotropic Rankine, (c) isotropic Drucker-Prager and (d) orthotropic Drucker-Prager

Figures 27 and 28 show the maximum principal stresses computed with standard and mixed FE, respectively. For isotropic damage, Figures $27 \mathrm{a}$ and $27 \mathrm{c}$, stresses concentrate at the tip of the crack causing it to progress while the material unloads in the crack path as the material is damaged. With standard FE and orthotropic damage, Figures $27 \mathrm{~b}$ and $27 \mathrm{~d}$, severe stress 
oscillations appear along the sides of the crack. With mixed FE, Figure 28 orthotropic models produce similar results to isotropic damage in terms of the stress field. No stress oscillations appear along the crack path. No trace of spurious stress transfer can be appreciated with the isotropic models, neither in the solution computed with standard or mixed FE, nor in the orthotropic solution computed with mixed FE. Contrariwise, severe spurious stresses are evident for the standard orthotropic damage along the crack path.

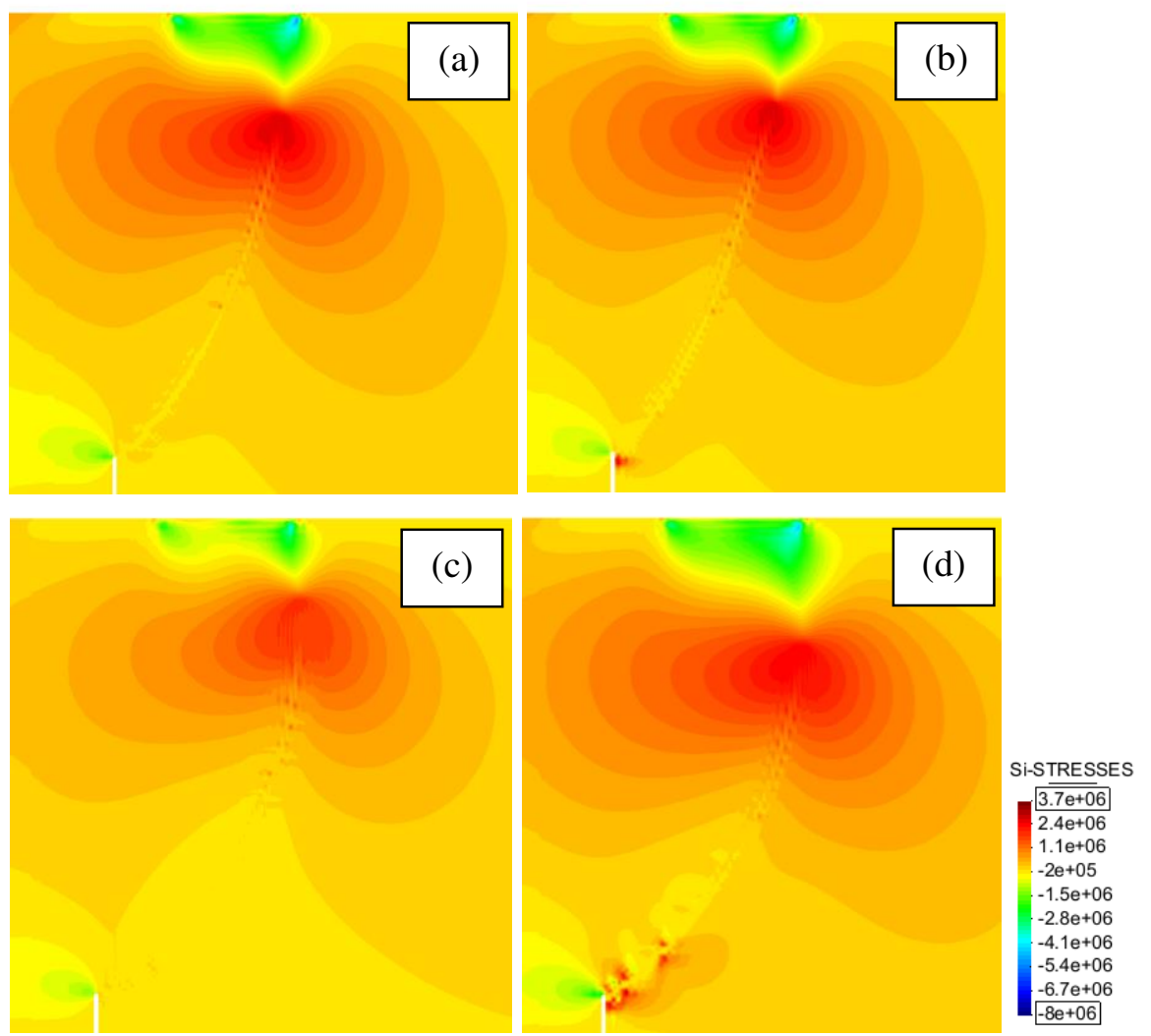

Figure 28. Maximum principal stress of the mixed mode bending test, set $\mathbf{C}$, computed with mixed FE with (a) isotropic Rankine, (b) orthotropic Rankine, (c) isotropic Drucker-Prager and (d) orthotropic Drucker-Prager

For the mixed formulation, results are very similar regardless of whether isotropic or orthotropic models are used, both in terms of the computed crack paths (Figures 16 and 19) and force-CMSD curves (Figures 18 and 21). This does not happen with standard FE, as can be seen in the crack paths of Figures 25 and 26 or in the computed force-CMSD curves of Figures 29 and 30. 

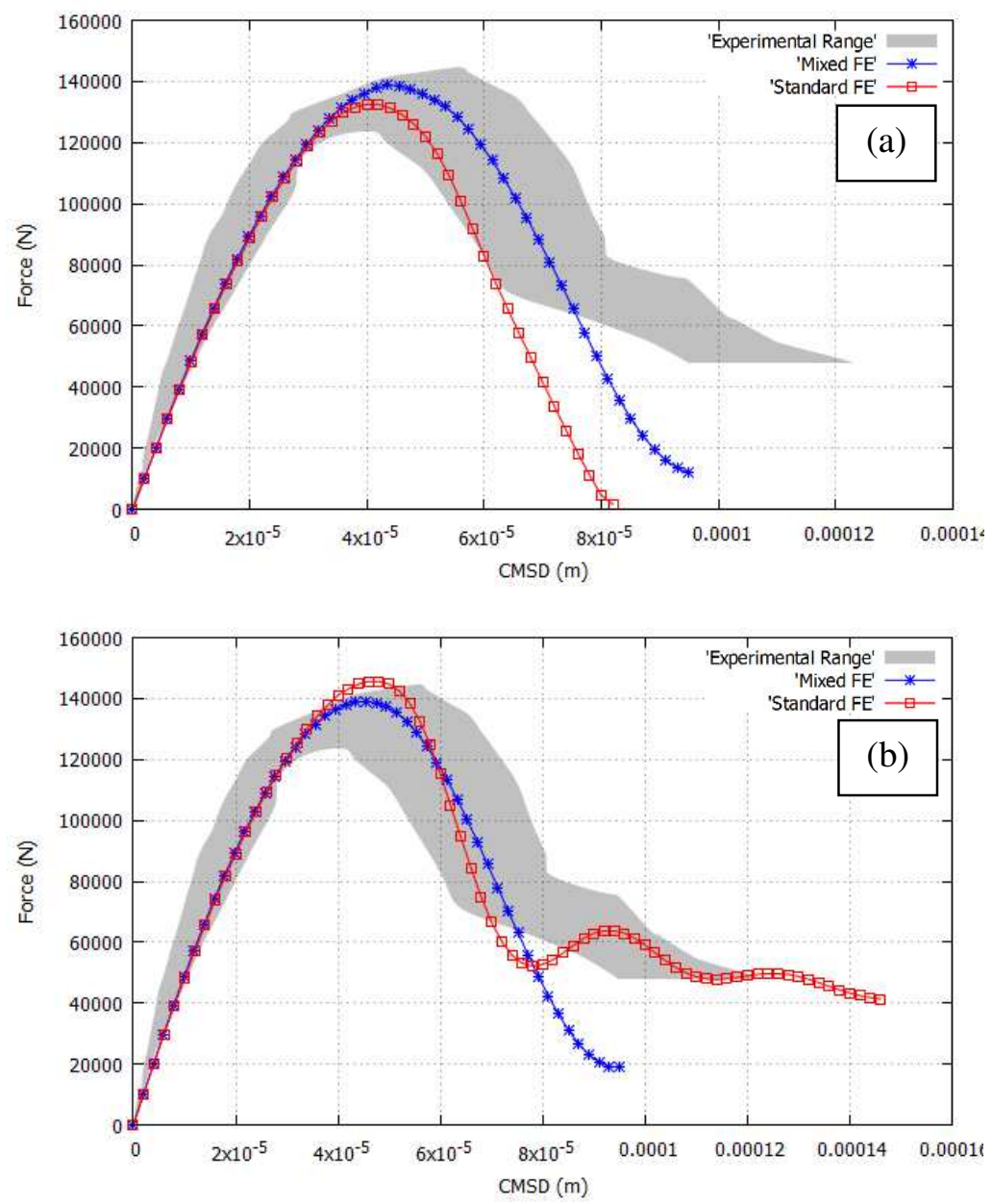

Figure 29. Force-CMSD curve of the mixed mode bending test for set $\mathrm{C}$ with (a) isotropic and (b) orthotropic Rankine models

Using isotropic damage models, the standard FE formulation is well-known to produce unrealistic mesh-biased results in many situations, so as to make it unreliable. Using orthotropic damage models, the discontinuous approximation of the strain field and the meagerness of the kinematic description of low order standard FE produces strain oscillations and spurious stresses that make their implementation unpractical.

The above problems are greatly alleviated by the enhanced kinematical description of the mixed $\boldsymbol{\varepsilon} / \boldsymbol{u}$ finite elements. 

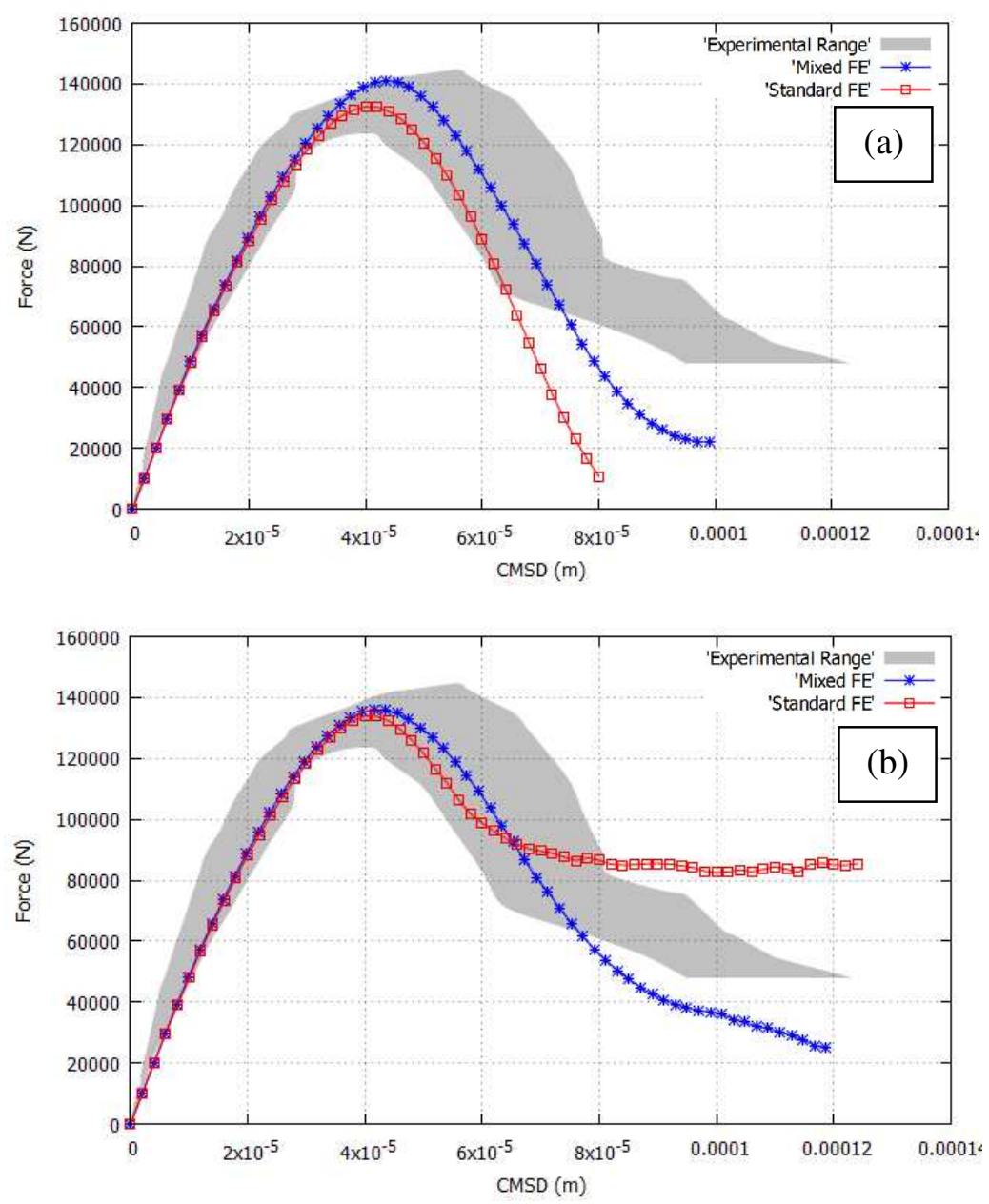

Figure 30. Force-CMSD curve of the mixed mode bending test for set $\mathrm{C}$ with (a) isotropic and (b) orthotropic Drucker-Prager models

\subsection{Mixed mode shearing-tension test}

The numerical analysis of a mixed mode shearing-tension test is considered. The specimen was experimentally tested by Nooru-Mohamed [44] and numerical simulations were made in references [45-50]. A tracking algorithm was considered in references [46-47, 49-50], while in [48] a sequentially linear analysis to non-proportional loading was done. In reference [45], the performance of several local and nonlocal models is addressed. 


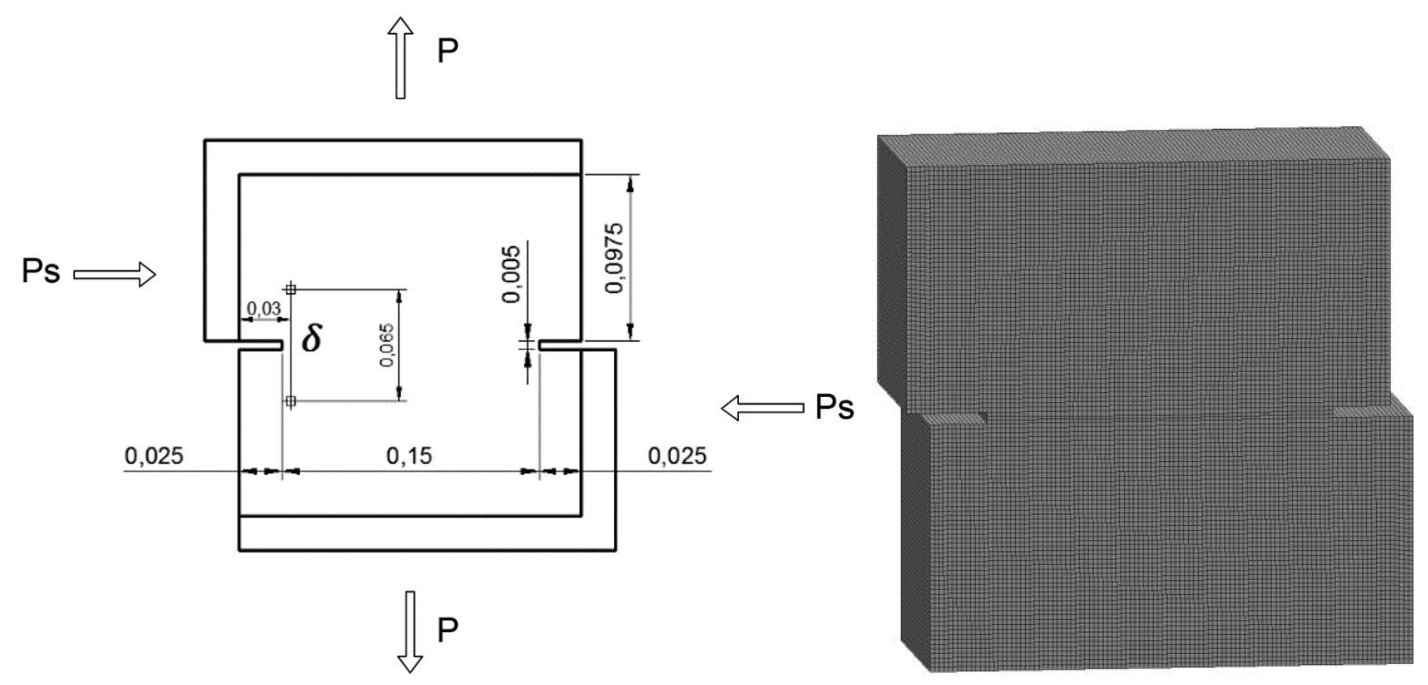

Figure 31. Geometry of the mixed mode shearing-tension test (m)

\begin{tabular}{|c|c|}
\hline Young's Modulus & $30.0 \cdot 10^{9} \mathrm{~Pa}$ \\
\hline Poisson's Ratio & 0.2 \\
\hline Tensile Strength & $3.0 \cdot 10^{6} \mathrm{~Pa}$ \\
\hline Fracture Energy & $60 \mathrm{~J} / \mathrm{m}^{2}$ \\
\hline Compressive Strength & $6.0 \cdot 10^{7} \mathrm{~Pa}$ \\
\hline
\end{tabular}

Table 6. Material properties of the mixed mode shearing-tension test

The objective of this example is to assess the performance of the Drucker-Prager criterion in computing mixed mode cracking.

The geometry of the specimen is shown in Figure 31. The thickness of the specimen is $0.05 \mathrm{~m}$. The loads are applied in two stages. In the first one, vertical tensile forces $P$ are kept to zero while the horizontal shear forces $P_{S}$ are incremented until a certain value. In the second stage, the loads $P_{S}$ are kept constant and the loads $P$ are incremented until failure occurs. The tests are performed under several loading conditions: in unit $4 \mathrm{a}, P_{S}$ reaches a value of $5 \mathrm{kN}$, in unit $4 \mathrm{~b} P_{S}$ gets to 10 $\mathrm{kN}$ and in $4 \mathrm{c}, 27.5 \mathrm{kN}$. The load is applied in the specimen through contact with a rigid steel frame. In the numerical simulation, the load is applied in the first stage as horizontal forces and in the second stage via increments of vertical displacements to accurately capture the post-peak behavior.

The simulation is performed using the isotropic and orthotropic Drucker-Prager damage constitutive models. The material properties for the mixed mode shearing-tension test are shown in Table 6.

For this example, 3D hexahedral elements are used, resulting in a fully structured mesh of 23,748 nodes and elements of $2 \mathrm{~mm}$, shown in Figure 31. 

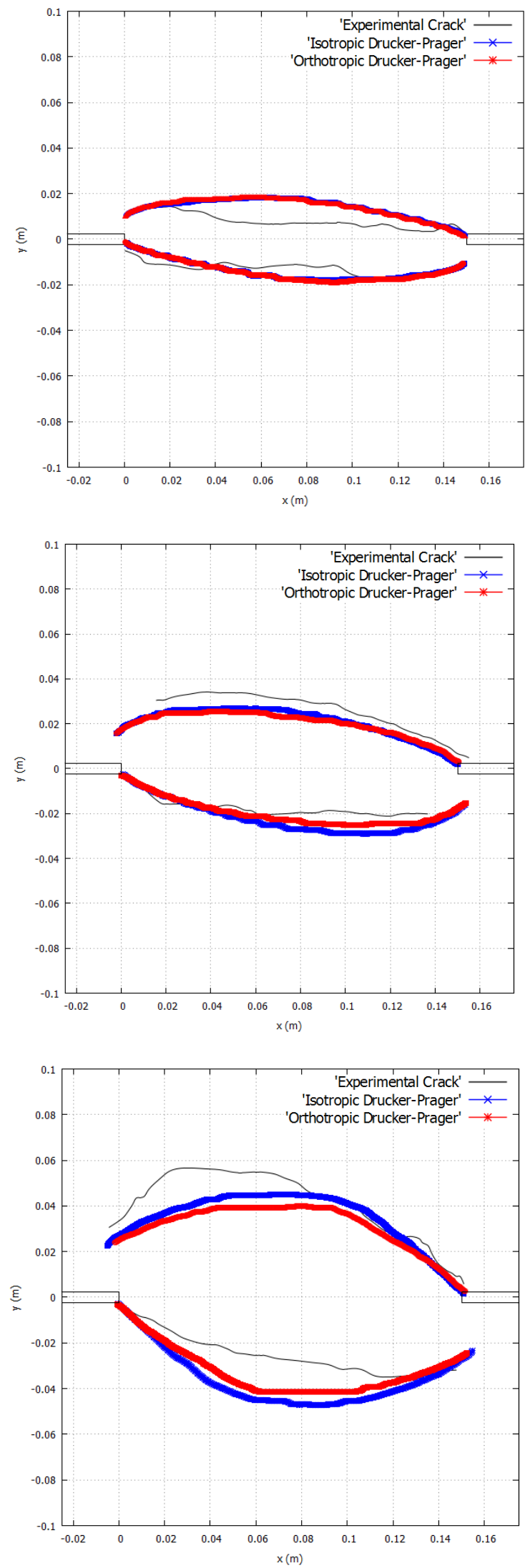

Figure 32. Crack trajectories of the mixed mode shearing-tension test, units $4 a, 4 b$ and $4 c$, for the isotropic and orthotropic Drucker-Prager models 

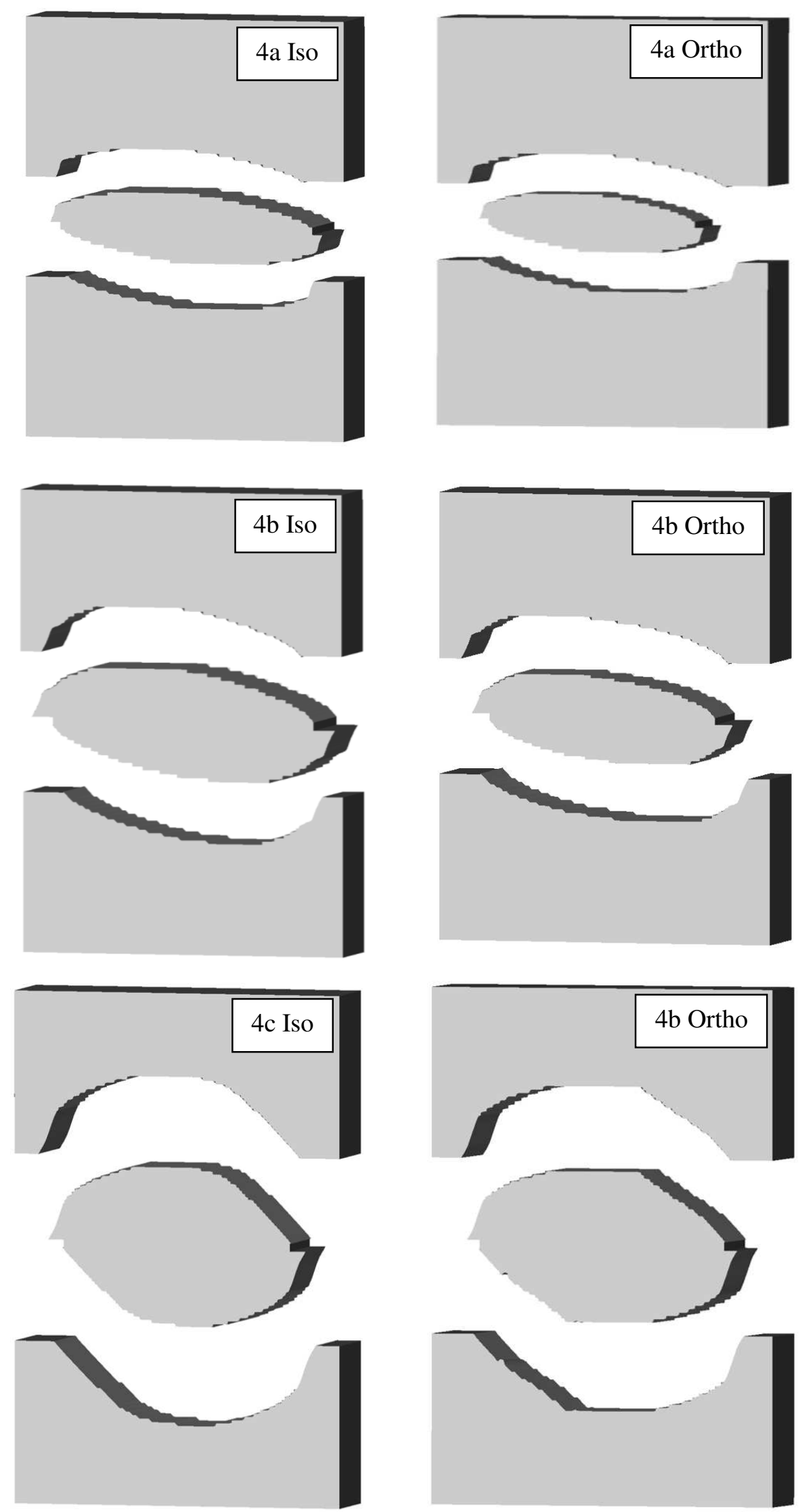

Figure 33. Crack surfaces of the mixed mode shearing-tension test, units $4 a, 4 b$ and $4 c$, for the isotropic and orthotropic Drucker-Prager models 

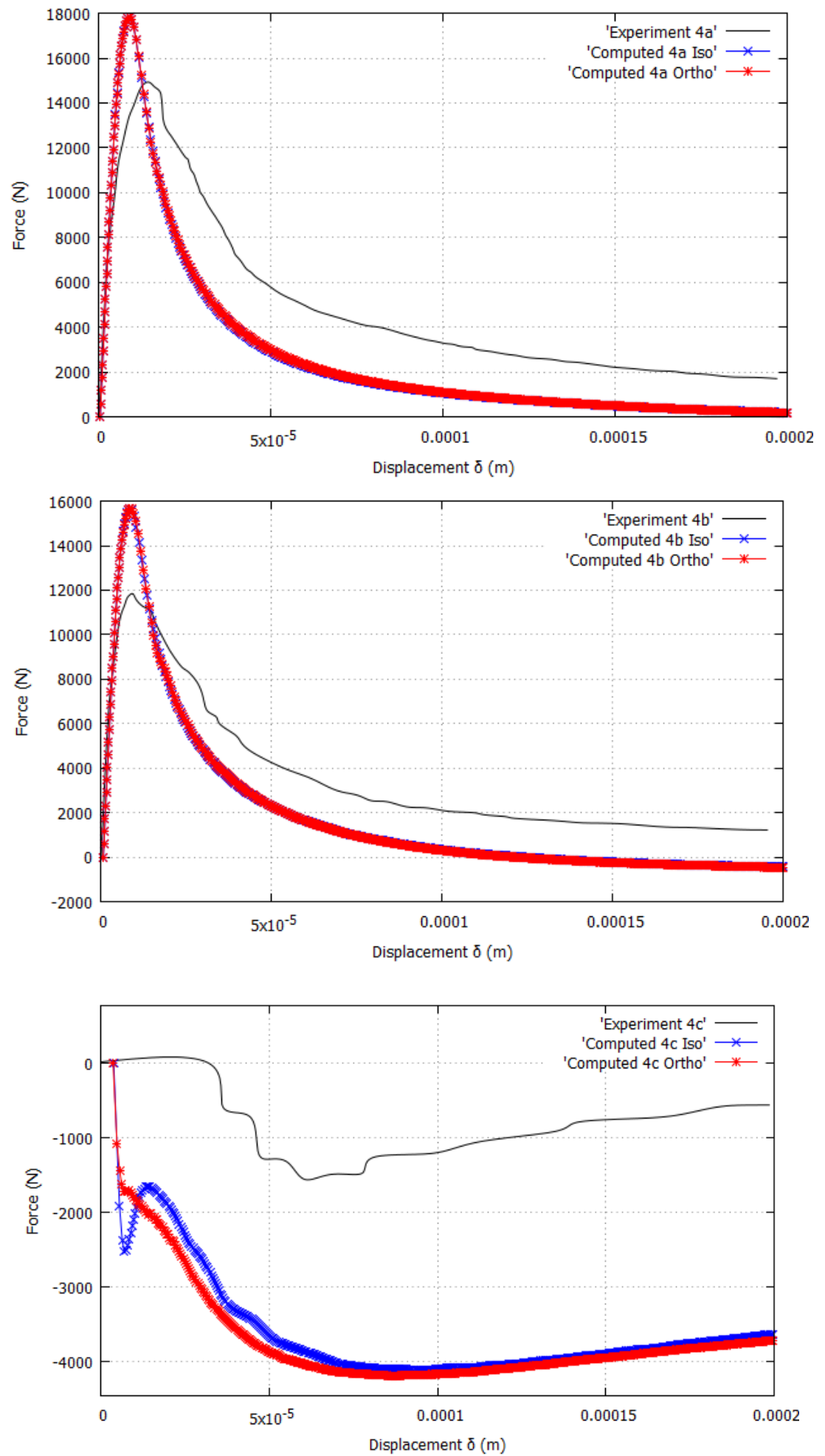

Figure 34. Force-displacement curves of the mixed mode shearing-tension test for units $4 a, 4 b$ and $4 c$ 


\section{Isotropic and orthotropic Drucker-Prager models}

Figure 32 shows the crack trajectories of the mixed mode shearing-tension test computed with the isotropic and orthotropic Drucker-Prager models for units $4 \mathrm{a}, 4 \mathrm{~b}$ and $4 \mathrm{c}$. The results computed with both the isotropic and orthotropic damage models are very similar. It can be seen that they are also rather resembling to the experiments for all the units. The computed results present the polar symmetric behavior expected for the intended loading and boundary conditions of the test. It should be noted that the experimental results lack this polar symmetry. There are several possible reasons for this, as it has been noted in [44]. Note that the computed crack paths are halfway between the upper and the lower cracks in the experiment.

Figure 33 also shows the crack surfaces resulting from the 3D analysis, with isotropic and orthotropic damage, plotted as an iso-level surface of the norm of displacements and the three pieces in which the specimen breaks. Both models produce lifelike crack surfaces.

Figure 34 shows the force- $\delta$ displacement curves for all three units. It can be seen that in all the specimens the experimental peak load is significantly lower than the computed one. This behavior has been systematically observed in all the solutions computed in references [47-50]. This may be due to the relative lack of symmetry of the experiment, resulting in slightly different crack paths and correspondingly different force-displacement curves. The isotropic and orthotropic models produce almost overlapping force- $\delta$ displacement curves.

In Scenario 4c, the axial force $P$ reverses sign, turning rapidly to be negative, even if the applied axial displacement is positive, corresponding to pulling apart of the fixing frames. This behavior is peculiar and rather difficult to capture in a numerical simulation. References [47,50] are able to capture it, while $[48,49]$ did not include this particular scenario. Figure 34 shows that the simulation captures the experimental behavior qualitatively, even if the quantitative difference is significant. Note that the value of the normal forces $P$ involved in this case is much lower than the shear forces $P_{S}$.

\subsection{Torsion test on a solid cylindrical specimen}

In this section, the numerical analysis of a cylindrical concrete specimen subjected to a torsional load is performed. This example has been included to assess the performance of several isotropic and orthotropic damage models in Mode III tearing crack failure.

The specimen is a cylinder $450 \mathrm{~mm}$ long and with a $50 \mathrm{~mm}$ radius. A small hole of $5 \mathrm{~mm}$ radius has been introduced in the middle of the specimen to fix the occurrence of fracture. The material properties of the test are shown in Table 7. The load $\mathrm{F}$ inducing torsion is applied via increments of vertical displacements as shown in Figure 35.

\begin{tabular}{|c|c|}
\hline Young's Modulus & $38 \cdot 10^{9} \mathrm{~Pa}$ \\
\hline Poisson's Ratio & 0.0 \\
\hline Tensile Strength & $2.3 \cdot 10^{6} \mathrm{~Pa}$ \\
\hline Fracture Energy & $80 \mathrm{~J} / \mathrm{m}^{2}$ \\
\hline Compressive Strength & $4.6 \cdot 10^{7} \mathrm{~Pa}$ \\
\hline
\end{tabular}

Table 7. Material parameters of the torsion test 


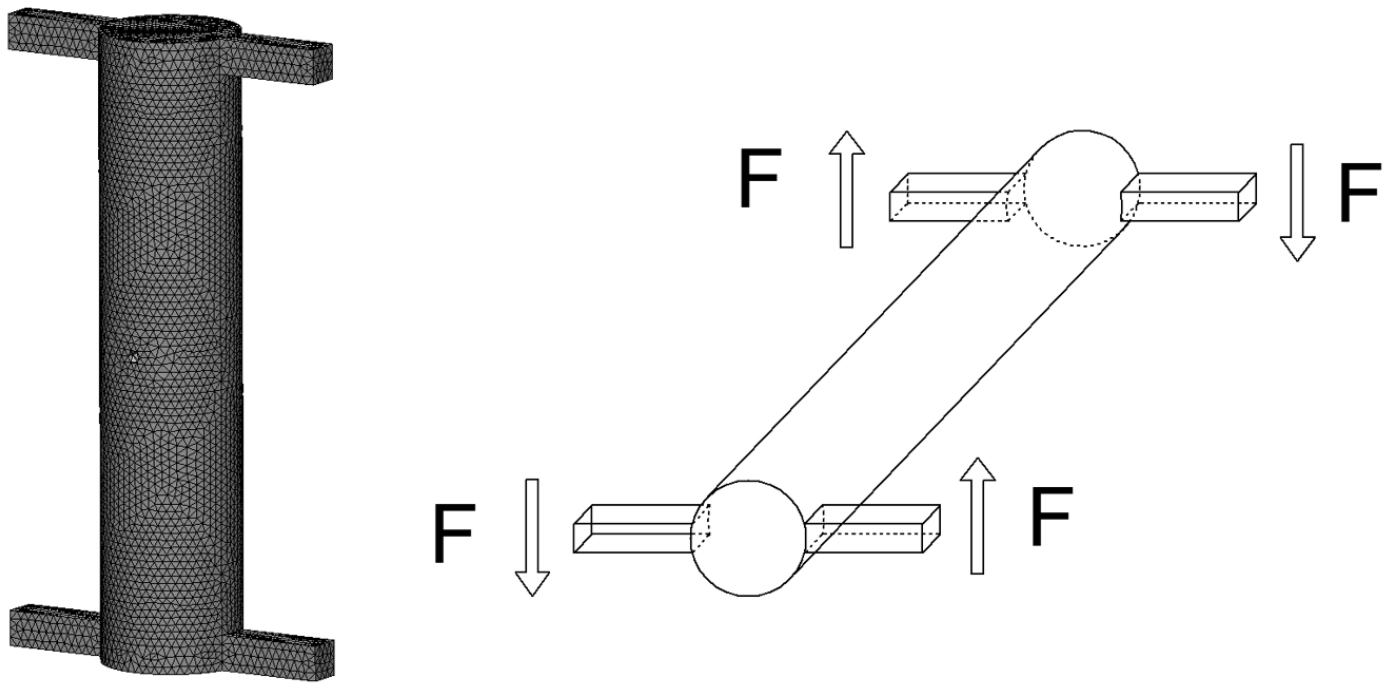

Figure 35. Geometry and loading of the torsion test (m)

To assess the performance of the different constitutive laws compared in this paper, the numerical results from the torsion test are compared to an analogous experiment, performed with a piece of chalk. The objective of this section is to accurately reproduce the twisting crack surface of the chalk shown in Figure 36, produced by pure torsional loading until failure.

Unlike for the case of uniaxial traction of section 4.2, the influence of Poisson's ratio in the solution of the three dimensional torsion test in terms of crack surface is negligible.

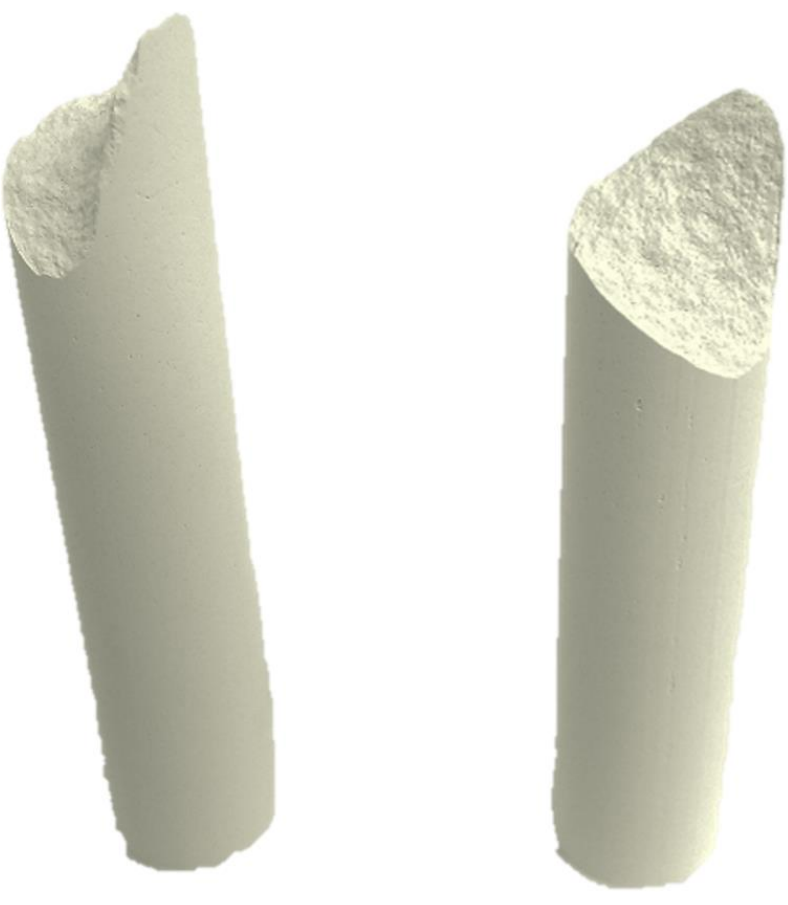

Figure 36. Analogous experiment of torsion test with a piece of chalk

This example is solved with the mixed FEM using 3D tetrahedral elements. The computational domain is discretized with fully unstructured meshes with elements of $6 \mathrm{~mm}$, resulting in a mesh of 22,518 nodes, shown in Figure 35. 


\section{Isotropic models}

Figure 37 (top) shows the computed crack surfaces in the specimen obtained with the isotropic Beltrami, Positive Beltrami, Rankine and Drucker-Prager criteria, plotted as the level set surface of the norm of displacements.

The Beltrami criterion produces a planar horizontal crack surface. This is accountable to the fact that, for pure torsion, this criterion coincides with the Von Mises criterion, that would yield exactly such a planar crack in the plane of maximum shear stress. Note that the Positive Beltrami and Rankine models produce very similar results, an helicoidal crack, which is explicable by their fairly similar formulation. As it has been explained in Section 2, these two criteria are only sensitive to tensile stress. Of all the isotropic models considered, the only one that is able to reproduce the helicoidal crack with $45^{\circ}$ slope that occurs in the piece of chalk is the DruckerPrager criterion.

\section{Orthotropic models}

Figure 37 (bottom) shows the computed crack surfaces obtained with the orthotropic Rankine and Drucker-Prager criteria. In this case, the isotropic and orthotropic Rankine models produce noticeably different results, even if they are driven by the same failure criteria. The reason for this is that the corresponding inelastic deformations are different. Only the orthotropic Rankine model produces the correct slope of $45^{\circ}$ for the helicoidal crack. The orthotropic Drucker-Prager model performs similarly to its isotropic counterpart.

All these orthotropic criteria produce slightly different crack surfaces, all similar to the experiment, but the one that reproduces better the actual crack of the chalk in Figure 36 is the orthotropic Rankine constitutive law, as can be seen in detail in Figure 38. There, the similarities of the computed crack surface using this criterion and the test performed with the piece of chalk are highlighted. 


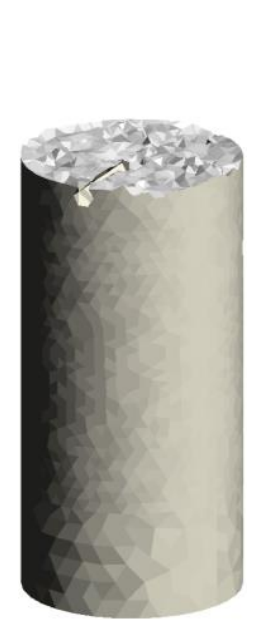

Isotropic Beltrami

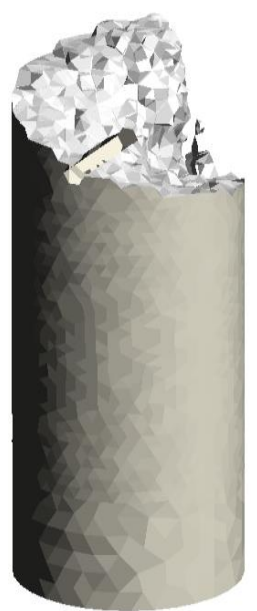

Isotropic Positive Beltrami

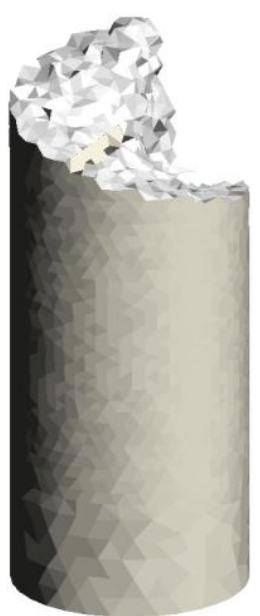

Isotropic Rankine

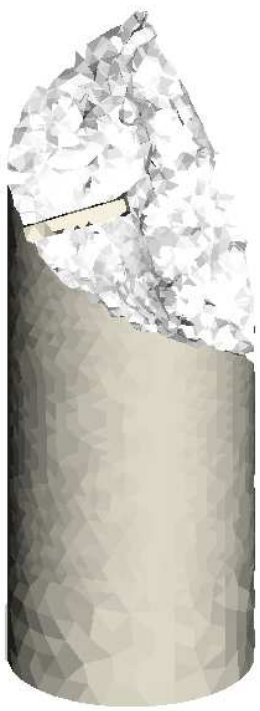

Isotropic Drucker-Prager

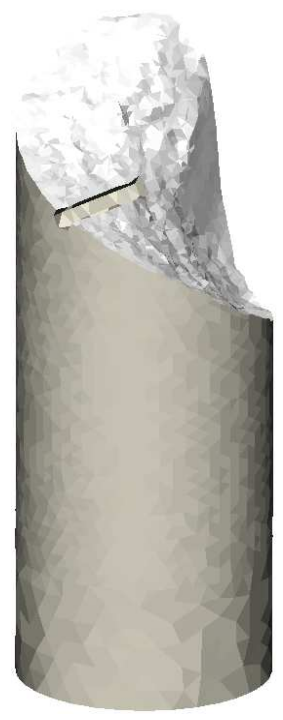

Orthotropic Rankine

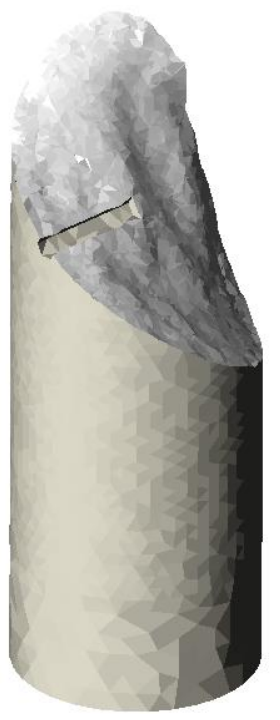

Orthotropic Drucker-Prager

Figure 37. Crack surfaces of the torsion test, with several failure criteria 

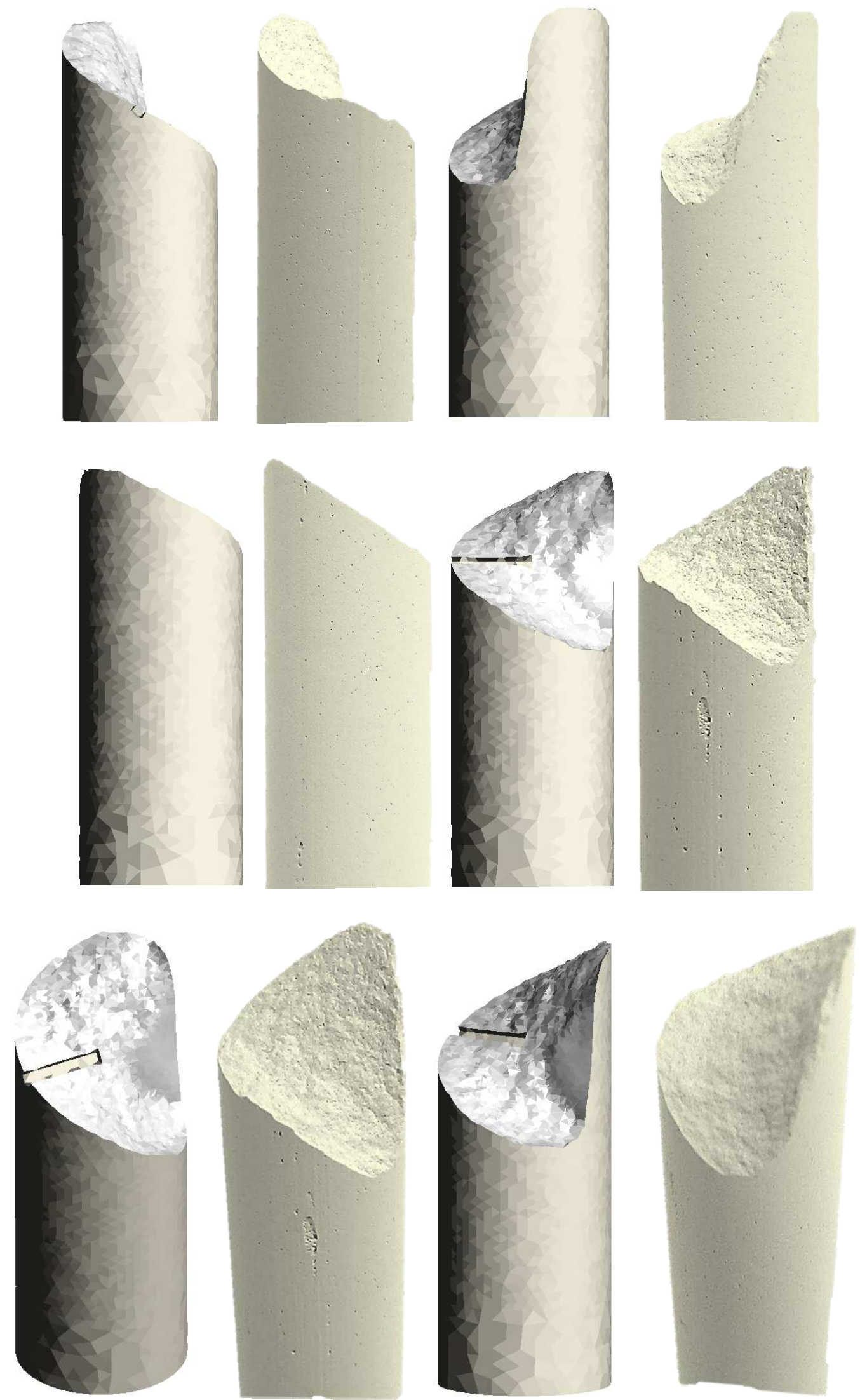

Figure 38. Different views of the crack surface obtained with the orthotropic Rankine damage model 


\section{Conclusions}

In this work, the appraisement of planar, bending and twisting cracks in 3D is performed. To this end, a mixed finite element formulation is considered and the performance of several isotropic and orthotropic damage models is assessed. Several simulations are compared to theoretical and experimental results reported in the literature.

It is concluded that:

- The mixed finite element formulation is appropriate for solving the problem of quasibrittle crack propagation without any mesh bias of the computed solution and without the need of any tracking technique.

- The enhanced accuracy of the mixed FE formulation allows for a transparent assessment of the several damage models considered without problems of spurious mesh bias associated to standard elements.

- The mixed FE formulation is fit to accommodate isotropic and orthotropic damage models.

- The enforcement of strain continuity in the mixed $\boldsymbol{\varepsilon} / \boldsymbol{u}$ FE formulation practically vanishes the strain oscillations, and corresponding spurious stress transfer, in the crack and its vicinity, that pollute solutions obtained with the standard FE formulation.

- Isotropic and orthotropic damage models are able to compute planar, bending and twisting cracks with enhanced accuracy, producing solutions that match the analytical results and experiments.

- Different damage models produce different solutions in terms of crack surface and forcedisplacement curves to the same problem (geometry and loading).

- Differences between isotropic and orthotropic models depend on the actual problem. In some cases, significant variations are observed in terms of crack surfaces or forcedisplacement curves, while in other cases the models behave similarly.

- In mode I loading, differences between the isotropic and orthotropic models are caused by the Poisson effect.

- In problems of mixed mode I and II loading, the Drucker-Prager criterion produces excellent results in terms of computed crack path and load vs displacement curves. For this type of loading isotropic and orthotropic models produce very similar results.

- In mode III fracture, the Rankine criterion is remarkable when used in its orthotropic form. In this case, all the orthotropic damage models yield similar results.

From these, it is confirmed that the mixed FE formulation largely overcomes the difficulties associated both to isotropic and orthotropic damage models when using in the standard FE framework. Orthotropic models can be extended to include microcrack closure-reopening (MCR) effects, so that cyclic loading can be taken into account.

It is also noteworthy that all the examples in this paper are solved in 3D and that both tetrahedral and hexahedral FE are used. Prism elements can be similarly used, as the proposed FE formulation is not restricted to a particular FE integration. 


\section{References}

[1] J. Rots, "Computational modeling of concrete fracture," Ph.D. Thesis, Delft University of Technology, 1988.

[2] M. Jirasek and T. Zimmermann, "Analysis of Rotating Crack Model," Journal of engineering mechanics, vol. 124, no. 8, pp. 842-851, 1998.

[3] Y. Rashid, "Ultimate strength analysis of prestressed concrete pressure vessels," Nuclear Engineering and Design, vol. 7, no. 4, pp. 334-344, 1968.

[4] R. Cope, P. Rao, L. Clark and P. Norris, "Modelling of reinforced concrete behavior for finite element analysis of bridge slabs," Numerical Methods for Nonlinear Problems, Pineridge Press, Swansey, pp. 457-470, 1980.

[5] Z. Bazant, "Comment on orthotropic models for concrete and geomaterials," Journal of Engineering Mechanics, vol. 3, no. 109, pp. 849-865, 1983.

[6] R. Litton, "A contribution to the analysis of concrete structures under cyclic loading," Ph.D. Thesis, University of California, Berkeley, 1976.

[7] R. de Borst and P. Nauta, "Non-orthogonal cracks in a smeared finite element model," Engng. Computation, vol. 2, pp. 35-46, 1985.

[8] Z. Bazant, "Size effect in blunt fracture: concrete, rock, metal," Journal of Engineering Mechanics, vol. 4, no. 110, pp. 518-535, 1984.

[9] I. Carol and P. Prat, "A statically constrained microplane model for the smeared analysis of concrete cracking," International Conference on Computer Aided Analysis and Design of Concrete Structures, vol. 2, pp. 919-930, 1990.

[10] K. Willam, E. Pramono and S. Sture, "Fundamental issues of smeared crack models," SEM/RILEM, International Conference on Fracture of Concrete and Rock, pp. 142-153, 1987.

[11] P. Feenstra, "Computational aspects of biaxial stress in plain and reinforced concrete," Ph.D. Thesis, Delft University of Technology, 1993.

[12] S. Weihe and B. Kroplin, "Fictitious crack models: a classification approach," Aedificatio publishers, Freiburg, 1995.

[13] S. Weihe, B. Kroplin and R. De Borst, "Classification of smeared crack modles based on material and structural properties," Int. J. Solids Structures, no. 12, pp. 1289-1308, 1998. 
[14] R. Faria, J. Oliver and M. Cervera, "A strain-based plastic viscous-damage model for massive concrete structures," International Journal of Solids and Structures, vol. 35, no. 14, pp. 1533-1558, 1998.

[15] G. Hofstetter and G. Meschke, "Numerical modeling of concrete cracking," Springer Science and Business Media, vol. 532, 2011.

[16] J. Oliver, M. Cervera, S. Oller and J. Lubliner, "Isotropic damage models and smeared crack analysis of concrete," II int. conference on computer aided analysis and design of concrete, 1990.

[17] M. Cervera, E. Hinton and O. Hassan, "Nonlinear analysis of reinforced concrete plate and shell structures using 20-noded isoparametric brick elements," Computers and Structures, vol. 25, no. 6, pp. 845-869, 1987.

[18] M. Cervera, "An orthotropic mesh corrected crack model," Computer Methods and Applied Mechanics in Engineering, vol. 197, no. 17-18, pp. 1603-1619, 2008.

[19] M. Cervera, "A smeared-embedded mesh-corrected damage model for tensile cracking," International Journal for Numerical Methods in Engineering, pp. 19301954, 2008.

[20] M. Cervera, M. Chiumenti and R. Codina, "Mixed stabilized finite element methods in nonlinear solid mechanics. Part I: Formulation," Computer Methods in Applied Mechanics and Engineering, vol. 199, no. 37-40, pp. 2559-2570, 2010.

[21] M. Cervera, M. Chiumenti and R. Codina, "Mixed stabilized finite element methods in nonlinear solid mechanics. Part II: Strain localization," Computer Methods in Applied Mechanics and Engineering, vol. 199, no. 37-40, pp. 2571-2589, 2010.

[22] M. Cervera and C. Tesei, "An energy-equivalent $d+/ d-$ damage model with enhanced microcrack closure-reopening capabilities for cohesive-frictional materials," Materials, vol. 10, no. 4, p. 433, 2017.

[23] M. Cervera, M. Chiumenti and R. Codina, "Mesh objective modelling of cracks using continuous linear strain and displacement interpolations," International Journal for Numerical Methods in Engineering, vol. 87, no. 10, pp. 962-987, 2011.

[24] M. Cervera, M. Chiumenti, L. Benedetti and R. Codina, "Mixed stabilized finite element methods in nonlinear solid mechanics. Part III: Compressible and incompressible plasticity," Computer Methods in Applied Mechanics and Engineering, vol. 285, no. 0, pp. 752-775, 2015.

[25] M. Cervera, G. Barbat and M. Chiumenti, "Finite element modelling of quasi-brittle cracks in 2D and 3D with enhanced strain accuracy," Computational Mechanics, vol. 60 , no. 5 , pp. 767-796, 2017.

[26] O. Zienkiewicz, R. Taylor and Z. Zhu, "The finite element method, Vol. 1," 7th edition, Amsterdam, Elsevier Butterworth-Heinemann, 1989. 
[27] J. Simo and J. Ju, "Strain- and stress-based continuum damage models. I: Formulation; II Compatational aspects.," Int. J. Solids Struct., no. 23, pp. 821-869, 1987.

[28] J.-Y. Wu and M. Cervera, "Strain localization analysis of elastic-damaging frictional-cohesive materials: Analytical results and numerical verification," Materials, vol. 10, no. 4, 2017.

[29] G. Voyiadjis, Z. Taqieddin and P. Kattan, "Anisotropic damage-plasticity model for concrete," Int. J. Plast., vol. 24, pp. 1946-1965, 2008.

[30] J. Courdebois and F. Sidoroff, "Endommagement anisotrope en elasticité et plasticité," J. Mec. Theor. Appl., vol. Special Volume, pp. 45-60, 1982.

[31] I. Carol, E. Rizzi and K. Willam, "On the formulation of anisotropic elastic degradation. I: Theory based on pseudo-logarithmic damage tensor rate; II: Generalized pseudo-Rankine model for tensile damage," Int. J. Solids Struct., vol. 38, no. 4, pp. 491-546, 2001.

[32] L. Benedetti, M. Cervera and M. Chiumenti, "3D modelling of twisting cracks under bending and torsion skew notched beams," submitted to Engineering Fracture Mechanics, 2017.

[33] M. Cervera, C. Agelet de Saracibar and M. Chiumenti, "COMET: Coupled Mechanical and Thermal Analysis. Data Input Manuel, Version 5.0, Technical report IT-308. Available from http://www.cimne.upc.edu," 2002.

[34] "GiD: the personal pre and post-processor.," CIMNE, Technical University of Catalonia, p. <http://gid.cimne.upc.ed>, 2002.

[35] J.-Y. Wu and S.-L. Xu, "An augmented multicrack elastoplastic damage model for tensile cracking," International Journal of Solids and Structures, vol. 48, pp. 25112528, 2011.

[36] P. Feenstra and R. de Borst, "A plasticity model and algorithm for mode-I cracking in concrete," Int. J. Numer. Methods Eng., vol. 38, no. 5, pp. 2509-2529, 1995.

[37] P. Pivonka, J. Ozbolt, R. Lackner and H. Mang, "Comparative studies of 3Dconstitutive models for concrete: application to mixed-mode fracture," Int. J. Numer. Methods Eng., vol. 60, pp. 549-570, 2004.

[38] M. Arrea and A. Ingraffea, "Mixed-mode crack propagation in mortar and concrete," Report No. 81-13, Department of Structural Engineering, Cornell University, Ithaca, NY, 1982.

[39] J. Galvez and D. Cendón, "Simulación de la fractura del hormigón en modo mixto," Rev. Int. Met. Num. Calc. Dis. Ing., vol. 18, no. 1, pp. 31-58, 2002. 
[40] P. Areias, T. Rabczuk and J. César de Sá, "A novel two-stage discrete crack method based on the screened Poisson equation and local mesh refinement," Comput. Mech., vol. 58, pp. 1003-1018, 2016.

[41] T. Rabczuk and T. Belytschko, "Cracking particles: a simplified meshfree method for arbitrary evolving cracks," International Journal for Numerical Methods in Engineering, vol. 61, pp. 2316-2343, 2004.

[42] A. Saleh and M. Aliabadi, "Crack growth analysis in concrete using boundary element method," Engineering Fracture Mechanics, vol. 51, no. 4, pp. 533-545, 1995.

[43] W. Gerstle and M. Xie, "FEM modeling of fictitious crack propagation in concrete," J. Eng. Mech., vol. 118, no. 2, pp. 416-434, 1992.

[44] M. Nooru-Mohamed, "Mixed-mode fracture of concrete: an experimental approach," Ph.D. Thesis, Delft University of Technology, 1992.

[45] M. Di Prisco, L. Ferrara, F. Meftah, J. Pamin, R. De Borst, J. Mazars and J. Reynouard, "Mixed mode fracture in plain and reinforcement concrete: some results on benchmark tests," International Journal of Fracture, vol. 103, pp. 127-148, 2000 .

[46] J. Oliver, A. Huespe, E. Samaniego and E. Chaves, "Continuum approach to the numerical simulation of material failure in concrete," Int. J. Numer. Anal. Meth. Geomech., vol. 28, pp. 609-632, 2004.

[47] M. Cervera and M. Chiumenti, "Smeared crack approach: back to the original track," Int. J. Numer. Anal. Meth. Geomech., vol. 30, pp. 1173-1199, 2006.

[48] M. Dejong, M. Hendriks and J. Rots, "Sequentially linear analysis of fracture under non-proportional loading," Engineering Fracture Mechanics, vol. 75, pp. 50425056, 2008.

[49] A. Slobbe, M. Hendriks and J. Rots, "Smoothing the propagation of smeared cracks," Engineering Fracture Mechanics, vol. 132, p. 147-168, 2014.

[50] S. Saloustros, L. Pela and M. Cervera, "A crack-tracking technique for localized cohesive-frictional damage," Engineering Fracture Mechanics, vol. 150, pp. 96$114,2015$. 